Philosophical Magazine B, 2000, Vol. 80, No. 12, 2143-2173

\title{
Statistical theory of finite Fermi systems with chaotic excited eigenstates
}

\author{
V. V. Flambaum $\dagger$ and G. F. GRIBAKIN $\ddagger$ \\ School of Physics, University of New South Wales, Sydney 2052, Australia
}

[Received 10 April 2000 and accepted 22 May 2000]

\begin{abstract}
A theory of strongly interacting Fermi systems of a few particles is developed. At high excitation energies (a few times the single-particle level spacing) these systems are characterized by an extreme degree of complexity due to strong mixing of the shell-model-based many-particle basis states by the residual twobody interaction. This regime can be described as many-body quantum chaos. Practically, it occurs when the excitation energy of the system is greater than a few single-particle level spacings near the Fermi energy. Physical examples of such systems are compound nuclei, heavy open shell atoms (e.g. rare earths) and multicharged ions, molecules, clusters and quantum dots in solids. The main quantity of the theory is the strength function which describes spreading of the eigenstates over many-particle basis states (determinants) constructed using the shell-model orbital basis. A nonlinear equation for the strength function is derived, which enables one to describe the eigenstates without diagonalization of the Hamiltonian matrix. We show how to use this approach to calculate mean orbital occupation numbers and matrix elements between chaotic eigenstates and introduce typically statistical variables such as temperature in an isolated microscopic Fermi system of a few particles.
\end{abstract}

\section{$\S 1$. INTRODUCTION}

As is known, quantum-statistical laws are derived for systems with infinite number of particles, or for systems in a heat bath, therefore, their applicability to isolated finite systems of a few particles is, at least, questionable. However, the density of many-particle energy levels increases extremely rapidly (typically, exponentially) with an increase in both the number of particles and the excitation energy. For this reason, even a weak interaction between particles can lead to strong mixing between large number of simple many-particle states, resulting in the so-called chaotic eigenstates. If the components of such eigenstates can be treated as random variables (onset of quantum chaos), statistical methods are expected to be valid even for an isolated dynamic system.

One should stress that statistical description of such isolated systems can be quite different from that based on standard canonical distributions; therefore, application of the famous Fermi-Dirac or Bose-Einstein formulae may give incorrect results. Moreover, for isolated few-particle systems a serious problem arises in the definition

$\dagger$ Email: flambaum@phys.unsw.edu.au.

$\ddagger$ Present address: Department of Applied Mathematics and Theoretical Physics, The Queen's University of Belfast, Belfast BT7 1NN, Northern Ireland. 
of temperature, or other thermodynamic variables such as entropy and specific heat (in comparison, in infinite systems, different definitions give the same result).

In this paper we outline a statistical theory of finite quantum systems of interacting particles based on generic statistical properties of chaotic eigenstates (the 'microcanonical' approach). Typical examples of such systems are compound nuclei, complex atoms, atomic clusters and isolated quantum dots. This work is based on a number of earlier publications (Flambaum 1993, 1994, Flambaum and Vorov 1993, Flambaum et al. 1994, 1996a, Gribakin et al. 1995, Flambaum and Izrailev 1997a,b), which developed different aspects of the theory.

\section{§2. STRENGTH FUNCTION OF CHAOTIC EIGENSTATES}

\subsection{Equation for the strength function}

In this section we obtain an equation for the strength function of chaotic eigenstates. It is derived for Hamiltonian matrices with random uncorrelated off-diagonal matrix elements. This equation generalizes the result obtained by Wigner (1955, 1957) for infinite random matrices with a linearly increasing diagonal and off-diagonal matrix elements equal to \pm 1 , randomly placed within a band of width $b$ along the diagonal. It also reproduces the equation for the strength function in sparse random matrices with a diffuse band derived by Fyodorov et al. (1996) using the supersymmetry method.

It is important that this equation can be used for calculating strength functions in real many-body systems where strong mixing is achieved owing to the residual twobody interaction between the particles. In this case, one usually starts with multiparticle basis states (determinants) constructed of single-particle orbitals obtained for a particular mean field, for example in the Hartree-Fock approximation:

$$
|k\rangle=\prod_{i=1}^{n} a_{\nu_{i}}^{\dagger}|0\rangle .
$$

We use Greek letters to enumerate the single-particle basis states (orbitals), and every basis state $k$ corresponds to a different set of $\nu_{1}, \ldots, \nu_{n}$. The matrix elements $H_{k_{j}}$ of the Hamiltonian

$$
\mathbf{H}=\sum_{\alpha} \varepsilon_{\alpha} a_{\alpha}^{\dagger} a_{\alpha}+\frac{1}{2} \sum_{\alpha \beta \gamma \delta} V_{\alpha \beta \gamma \delta} a_{\alpha}^{\dagger} a_{\beta}^{\dagger} a_{\gamma} a_{\delta},
$$

calculated with respect to this basis are correlated, even if the residual two-body interaction $V_{\alpha \beta \gamma \delta}$ is random (Flambaum et al. 1996a, Brody et al. 1981). However, the effect of these two-body correlations on the strength functions is small (see below).

Consider an unperturbed system which is described by a diagonal Hamiltonian matrix with some regular diagonal matrix elements:

$$
H_{k j}^{(0)}=E_{k} \delta_{k j} \text {. }
$$

This Hamiltonian describes a many-body system of non-interacting particles, or a system of interaction particles in the mean-field basis, if we neglect the residual twobody interaction. It is often convenient to enumerate the basis states in such a way that their mean energies $E_{k}=\langle k|\mathbf{H}| k\rangle$ increase with increasing $k$. The eigenstates of the system 


$$
\mathbf{H}|i\rangle=E^{(i)}|i\rangle
$$

expanded in terms of the basis states $k$,

$$
|i\rangle=\sum_{k} C_{k}^{(i)}|k\rangle,
$$

are characterized by their components ('wavefunctions') $C_{k}^{(i)}$, that is eigenvectors of the Hamiltonian matrix

$$
\sum_{j} H_{k j} C_{k}^{(i)}=E^{(i)} C_{k}^{(i)}
$$

We consider cases when mixing of the basis components due to the off-diagonal matrix elements $V_{k j} \equiv H_{k j}$ is strong. This regime is the opposite of perturbation theory. Hence, $V_{k j} \gg D$ must be fulfilled, where $D$ is the mean spacing between the diagonal 'energies' $E_{k}$ (see $\S 2.4$ ). Besides this, the off-diagonal matrix elements $V_{k j}$ in complex many-body systems appear to behave as random variables with zero mean $\overline{V_{k j}}=0$ (Flambaum et al. 1994, Gribakina et al. 1995, Horoi et al. 1995a,b, Frazier et al. 1996, Zelevinsky et al. 1996). In this situation the eigenstates (5) contain large numbers $N_{\mathrm{p}}$ of essentially non-zero 'principal' components of similar sizes $C_{k}^{(i)} \sim 1 / N_{\mathrm{p}}^{1 / 2}$, due to normalization $\sum_{i}\left|C_{k}^{(i)}\right|^{2}=\sum_{k}\left|C_{k}^{(i)}\right|^{2}=1$. If $D \neq 0$ and the Hamiltonian matrix is large, these components are centred around the corresponding eigenvalue $E^{(i)}$, within some characteristic energy interval $\left|E_{k}-E^{(i)}\right| \lesssim \Gamma$, where $\Gamma$ is the so-called spreading width. Its magnitude depends on the strength of the mixing interaction $V_{k j}$. This picture allows for variation of both $D$ and $\overline{V_{k j}^{2}}$ along the matrix, that is, as functions of the energy of the basis states. In real systems this variation is slow and smooth.

Because of the strong mixing the components $C_{k}^{(i)}$ of complicated (chaotic) eigenstate fluctuate almost randomly as functions of $k$ and $i$. However, these fluctuations aside, nearby eigenstates separated by energy intervals $\Delta E \sim D \ll \Gamma$ look similar. Therefore, one can study the smooth envelope of the weights $\left|C_{k}^{(i)}\right|^{2}$. This idea is realized in the strength function, also referred to as the local density of states

$$
\rho_{\mathrm{W}}(E, k)=\sum_{i}\left|C_{k}^{(i)}\right|^{2} \delta\left(E-E^{(i)}\right)
$$

introduced by Wigner. It shows how various basis states $k$ contribute to the eigenstates at energy $E$. A standard way to calculate $\rho_{\mathrm{W}}(E, k)$ is through the Green's function

$$
\mathbf{G}(E)=\frac{1}{E-\mathbf{H}+\mathrm{i} \eta}=\sum_{i} \frac{|i\rangle\langle i|}{E-E^{(i)}+\mathrm{i} \eta},
$$

or in the matrix form, using the eigenstate components:

$$
G_{k j}(E)=\sum_{i} \frac{C_{k}^{(i)} C_{j}^{(i)^{*}}}{E-E^{(i)}+\mathrm{i} \eta},
$$

where $\eta$ is positive infinitesimal. The usual rule $\left(E-E^{(i)}+\mathrm{i} \eta\right)^{-1}=$ $\left(E-E^{(i)}\right)^{-1}-\mathrm{i} \pi \delta\left(E-E^{(i)}\right)$ combined with the definition (7) then gives the key relation 


$$
\rho_{\mathrm{W}}(E, k)=-\frac{1}{\pi} \operatorname{Im}\left[G_{k k}(E)\right]
$$

In a similar way, one also finds the eigenvalue density (or level density)

$$
\begin{aligned}
\rho(E) & \equiv \sum_{i} \delta\left(E-E^{(i)}\right) \\
& =\sum_{i, k}\left|C_{k}^{(i)}\right|^{2} \delta\left(E-E^{(i)}\right)=\sum_{k} \rho_{\mathrm{W}}(E, k) \\
& =-\frac{1}{\pi} \operatorname{Im}\left(\sum_{k} G_{k k}(E)\right)=-\frac{1}{\pi} \operatorname{Im}\{\operatorname{Tr}[\mathbf{G}(E)]\} .
\end{aligned}
$$

The Green's function G, (equation (8)) can be presented as a perturbation theory expansion in powers of $\mathbf{V}$, the off-diagonal part of the Hamiltonian $\mathbf{H}=\mathbf{H}^{(0)}+\mathbf{V}$ :

$$
\begin{aligned}
\mathbf{G} & =\frac{1}{E-\mathbf{H}^{(0)}-\mathbf{V}+\mathrm{i} \eta} \\
& =\mathbf{G}^{(0)}+\mathbf{G}^{(0)} \mathbf{V} \mathbf{G}^{(0)}+\mathbf{G}^{(0)} \mathbf{V} \mathbf{G}^{(0)} \mathbf{V} \mathbf{G}^{(0)}+\cdots,
\end{aligned}
$$

where

$$
\mathbf{G}^{(0)}=\frac{1}{E-H^{(0)}+\mathrm{i} \eta} \quad \text { or } \quad G_{k j}^{(0)}=\frac{\delta_{k j}}{E-E_{k}+\mathrm{i} \eta}
$$

is the Green's function of the unperturbed Hamiltonian. In the matrix form, expansion (15) looks like

$$
G_{k j}=\frac{\delta_{k j}}{\mathcal{E}-E_{k}}+\frac{V_{k j}}{\left(\mathcal{E}-E_{k}\right)\left(\mathcal{E}-E_{j}\right)}+\sum_{m} \frac{V_{k m} V_{m j}}{\left(\mathcal{E}-E_{k}\right)\left(\mathcal{E}-E_{m}\right)\left(\mathcal{E}-E_{j}\right)}+\cdots,
$$

where we have introduced $\mathcal{E} \equiv E+$ i $\eta$ for the energy shifted off the real axis.

To obtain a smooth strength function averaged over the level-to-level fluctuations of the eigenstate components we should average the Green's function (15) over the random off-diagonal matrix elements $V_{k j}$. In doing so we assume that they have zero mean and are uncorrelated:

$$
\overline{V_{k j}}=0, \quad \overline{V_{k j} V_{l m}}=\overline{V_{k j}^{2}}\left(\delta_{k l} \delta_{j m}+\delta_{k m} \delta_{j l}\right) .
$$

We can also assume that, if the system is not placed in a magnetic field, the phases of the basis states can be chosen so that $V_{k j}=V_{j k}$ are real. The variance $\overline{V_{k j}^{2}}$ is a local average. It does not have to be constant over the matrix. On the contrary, in physical systems, owing to the oscillatory behaviour of the single-particle orbitals the mixing matrix elements should decrease when distant basis states are considered:

$$
\overline{V_{k j}^{2}} \rightarrow 0 \text { for }|k-j| \rightarrow \infty .
$$

Therefore, larger matrix elements are found along the main diagonal, and the Hamiltonian matrices are often modelled by banded (random) matrices (with $V_{k j} \neq 0$ only for $|k-j| \leqslant b$ ). Feature (19) also ensures that various sums containing products of $V_{k j}$ converge, even if the size of the Hilbert space is infinite. It is important that, while we assume averaging in the course of our derivation, it does not have to be performed explicitly in the final equations. There it is done automatically owing to summation over large numbers of basis states. 
The strength function is given by the diagonal matrix element $G_{k k}$ (equation (10)). Because of the random signs of $V_{k j}$, only terms with even powers of $\mathbf{V}$ in the perturbation expansion (15) survive after averaging:

$$
\begin{aligned}
G_{k j}= & \frac{1}{\mathcal{E}-E_{k}}+\sum_{m} \frac{V_{k m} V_{m k}}{\left(\mathcal{E}-E_{k}\right)\left(\mathcal{E}-E_{m}\right)\left(\mathcal{E}-E_{k}\right)} \\
& +\sum_{m, l, n} \frac{\frac{V_{k m} V_{m l} V_{l n} V_{n k}}{\left(\mathcal{E}-E_{k}\right)\left(\mathcal{E}-E_{m}\right)\left(\mathcal{E}-E_{l}\right)\left(\mathcal{E}-E_{n}\right)\left(\mathcal{E}-E_{k}\right)}+\cdots}{}
\end{aligned}
$$

It is convenient to represent this expansion graphically:

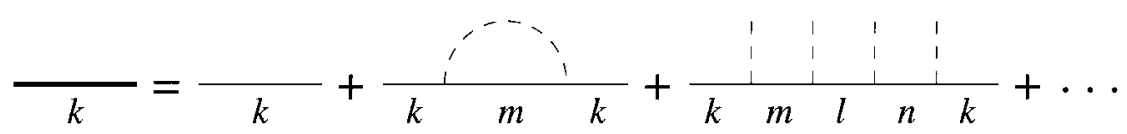

where the thick line is $G$, the thin lines are $G^{(0)}$, and the broken lines correspond to the matrix elements $V$. The semicircle broken line describes a binary product, for example $V_{k m} V_{m k}$ in the second-order diagram, which does not vanish upon averaging.

In the fourth order the non-zero contributions are given by the following contractions or binary associations:

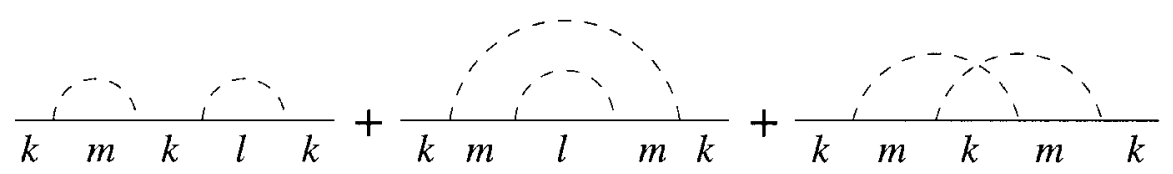

The first of them reduces to the product of the two second-order diagrams:

$$
\frac{1}{\mathcal{E}-E_{k}} \sum_{m} \frac{V_{k m}^{2}}{\left(\mathcal{E}-E_{m}\right)} \frac{1}{\mathcal{E}-E_{k}} \sum_{l} \frac{V_{k l}^{2}}{\left(\mathcal{E}-E_{l}\right)} \frac{1}{\mathcal{E}-E_{k}} .
$$

The second is irreducible; it does not contain $G_{k k}^{(0)}=\left(\mathcal{E}-E_{k}\right)^{-1}$ anywhere in the middle. Analytically it is given by

$$
\frac{1}{\mathcal{E}-E_{k}} \sum_{m, l} \frac{V_{k m}^{2} V_{m l}^{2}}{\left(\mathcal{E}-E_{m}\right)^{2}\left(\mathcal{E}-E_{l}\right)} \frac{1}{\mathcal{E}-E_{k}} .
$$

If we compare it with the expression for the third diagram in equation (22),

$$
\frac{1}{\mathcal{E}-E_{k}} \sum_{m} \frac{V_{k m}^{4}}{\left(\mathcal{E}-E_{m}\right)^{2}\left(\mathcal{E}-E_{k}\right)} \frac{1}{\mathcal{E}-E_{k}},
$$

we immediately see that it has a lower order of summation, that is it is given by a single sum over $m$, whereas in the other two the summation runs over $m$ and $l$. Since we consider systems in the strong-mixing regime, $\left|V_{k m}\right| \gg D$, every basis state is essentially coupled to many other basis states. Therefore, the sums over $m$ or $l$ in equations (23) and (24) and the diagram with the intersecting broken lines in equation (22) (equation (25)), is parametrically smaller than the other two. This 
rule is valid in all orders of the perturbation theory. For example, among the sixthorder diagrams, only the following five diagrams must be taken into account:

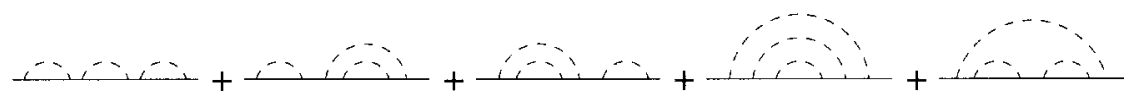

The diagrams with intersections

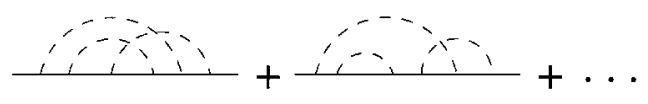

should be discarded.

Let us introduce the self-energy $\Sigma_{k}$ as a sum of all irreducible diagrams (without intersections), which in our case are also diagonal with respect to the end indices:

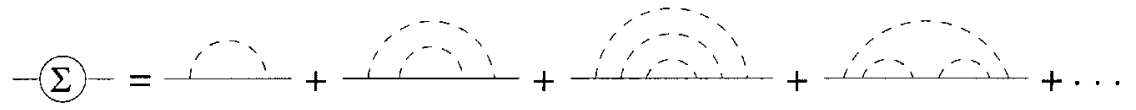

Similarly to the standard field-theory procedure, the Green's function can now be shown as

$$
\frac{}{k}=\frac{}{k}+\frac{\Sigma}{k} \sum_{k}+\frac{\Sigma_{k}}{\Sigma_{k}}+\cdots
$$

This geometric series immediately sums into the final expression for the Green's function

$$
G_{k k}(E)=\frac{1}{E-E_{k}-\Sigma_{k}(E)} .
$$

However, the most important consequence of the absence of the diagrams with intersections in $\Sigma_{k}$ is that series (28) can be presented as

$$
{ }_{k}(\Sigma)_{k}=\frac{i}{k m b}+\frac{i}{k m}(\Sigma)_{m k}+\frac{i}{k m}(\Sigma)_{m k}+\cdots \equiv \frac{i}{k \frac{1}{m}}
$$

which corresponds to the following equation:

$$
\Sigma_{k}(E)=\sum_{m} \frac{V_{k m}^{2}}{E-E_{m}-\Sigma_{m}(E)+\mathrm{i} \eta} .
$$

This nonlinear equation can be solved iteratively. Once $\Sigma_{k}(E)$ is found, the strength function is obtained from equations (10) and (30) as

$$
\rho_{\mathrm{W}}(E, k)=-\frac{1}{\pi} \operatorname{Im}\left(\frac{1}{E-E_{k}-\Sigma_{k}(E)}\right) .
$$


Equations (32) and (33) solve the problem of finding the strength function for a Hamiltonian with random off-diagonal matrix elements $\dagger$, and the total density of states is given by

$$
\rho(E)=-\frac{1}{\pi} \operatorname{Im}\left(\sum_{k} \frac{1}{E-E_{k}-\Sigma_{k}(E)}\right) .
$$

Note that the strength function (33) can be written as a Breit-Wigner formula

$$
\rho_{\mathrm{W}}(E, k)=\frac{1}{2 \pi} \frac{\Gamma_{k}}{\left(E-E_{k}-\Delta_{k}\right)^{2}+\Gamma_{k}^{2} / 4},
$$

where $\Gamma_{k}=-2 \operatorname{Im}\left[\Sigma_{k}(E)\right]$ is the energy (spreading) width of the basis component and $\Delta_{k}=\operatorname{Re}\left[\Sigma_{k}(E)\right]$ is the shift of the centre of the eigenvector with respect to the basis state energy $E_{k}$. Since both $\Gamma_{k}$ and $\Delta_{k}$ are in general energy dependent, the real shape of the strength function may be quite different from the simple Breit-Wigner profile with constant parameters.

\subsection{Particular cases}

\subsubsection{Full uniform random matrices}

If the off-diagonal matrix elements $V_{k m}$ are distributed uniformly over the matrix, $\overline{V_{k m}^{2}}=\mathbf{V}^{2}$, then $\Sigma_{k}(E)$ does not depend on $k$ and we have

$$
\begin{aligned}
\Sigma(E) & =\mathbf{V}^{2} \sum_{m} \frac{1}{E-E_{m}-\Sigma(E)+\mathrm{i} \eta}, \\
\rho(E) & =-\frac{1}{\pi \mathbf{V}^{2}} \operatorname{Im}[\Sigma(E)],
\end{aligned}
$$

which reproduces the result obtained by Pastur $(1972,1973)$ for $\mathbf{H}=\mathbf{H}^{(0)}+\mathbf{V}$, where $\mathbf{V}$ belongs to the Gaussian orthogonal ensemble.

\subsubsection{Sparse banded random matrices}

Fyodorov et al. (1996) considered the strength functions for large (infinite) matrices with equally spaced diagonal matrix elements, $E_{k}=k D$, and a sparse and banded random off-diagonal part. They chose the following probability distribution of $V_{\mathrm{km}}$ :

$$
P\left(V_{k m}\right)=\left(1-p_{k m}\right) \delta\left(V_{k m}\right)+p_{k m} h_{1}\left(V_{k m}\right),
$$

where $p_{k m}$ is the probability to find a non-zero matrix element at $(k, m)$. It was parametrized as $p_{k m}=(M / b) f(|k-m| / b)$, where $f$ is a decreasing function normalized as $b^{-1} \sum_{r=0}^{\infty} f(r / b)=1, b$ characterizes the width of the band, $M$ is the number of non-zero matrix elements in a row, and the probability density $h_{1}$ satisfies $\int h_{1}(v) \mathrm{d} v=1, \int v h_{1}(v) \mathrm{d} v=0$ and $\int v^{2} h_{1}(v) \mathrm{d} v=V^{2}$. With these definitions the locally averaged square of the off-diagonal matrix element is given by

$\dagger$ The authors are aware that a similar derivation was made in 1993 by O. K. Vorov (1993, unpublished). Its idea is also analogous to that of Edwards and Warner (1980), including the use of diagrams, although the latter considered the case where $\overline{V_{k j}^{2}}$ are constant over the matrix. 


$$
\overline{V_{k m}^{2}}=\frac{M}{b} f\left(\frac{|k-m|}{b}\right) \mathbf{V}^{2} .
$$

If we use this formula in equation (32), replace summation over $m$ by integration over $\mathrm{d} E_{m} / D$, take into account that $\Sigma_{k}(E)$ now depends only on $E-E_{k}$ and introduce new variables $x$ and $u$ as

$$
x=\frac{E-E_{k}}{R}, \quad u=\frac{E-E_{m}}{R},
$$

using $R^{2}=M \mathbf{V}^{2}$, the complex conjugate of equation (32) will be cast in the following form:

$$
g(x)=-\frac{1}{\kappa} \int_{\infty}^{\infty} \frac{f(|u-x| / \kappa) \mathrm{d} u}{\mathrm{i} u-g(x)},
$$

where $\kappa=D b / R$, and instead of $\Sigma_{k}$ a new function has been introduced by $\mathrm{i}\left(\Sigma_{k}\right)^{*} \equiv \operatorname{Rg}(x)$. Accordingly, the strength function as a function of $z=E-E_{k}$ is now given by

$$
\rho_{\mathrm{W}}(z)=\frac{1}{\pi} \operatorname{Re}\left(\frac{1}{\mathrm{i} z-\operatorname{Rg}(z / R)}\right) .
$$

Equations (39) and (40) were obtained by Fyodorov et al. (1996) by means of a technically involved method based on integration over commuting and anticommuting variables.

\subsubsection{Wigner random matrices}

In the pioneering work of Wigner $(1955,1957)$ the equations for the strength functions were obtained for random matrices with a regular diagonal $D=1$ and offdiagonal matrix elements $V_{k m}= \pm V$, placed randomly within the band $|k-m| \leqslant b$. If we use new variables,

$$
\frac{E-E_{k}}{b}=z, \quad \frac{E-E_{m}}{b}=\zeta,
$$

replace the summation over $m$ in equation (32) by integration over $\zeta$ and introduce the new function $p_{1}(z)=\mathrm{i} \Sigma_{k}(E) / \mathbf{V}^{2}$ and parameter $q=\mathbf{V}^{2} / b$, the strength function will be given by the following two equations:

$$
\begin{gathered}
\rho_{\mathrm{W}}(z)=\frac{1}{\pi b} \operatorname{Re}\left(\frac{\mathrm{i}}{z+\mathrm{i} q p_{1}(z)}\right), \\
p_{1}(z)=\int_{z-1}^{z+1} \frac{\mathrm{i}}{\zeta+\mathrm{i} q p_{1}(\zeta)} \mathrm{d} \zeta,
\end{gathered}
$$

which constituted the main result obtained by Wigner $(1955,1957)$.

\subsection{Exact solutions}

\subsubsection{Semicircle}

Suppose that the Hamiltonian is a full uniform random $N \times N$ matrix with $\overline{V_{k m}^{2}}=\mathbf{V}^{2}$, and the basis states are degenerate: $E_{k}=0$. In this case they are characterized by the same $\Sigma_{k}(E) \equiv \Sigma(E)$, which satisfies equation (32) with $E_{m}=0$ : 


$$
\Sigma(E)=\frac{(N-1) \mathbf{V}^{2}}{E-\Sigma(E)+\mathrm{i} \eta},
$$

which gives $\Sigma(E)=\frac{1}{2}\left[E-\mathrm{i}\left(4 N \mathbf{V}^{2}-E^{2}\right)^{1 / 2}\right]$, where we used $N-1 \approx N$ for $N \gg 1$. Substitution in equations (33) and (34) gives the strength function

$$
\rho_{\mathrm{W}}(E)=\frac{\left(4 N \mathbf{V}^{2}-E^{2}\right)^{1 / 2}}{2 \pi N \mathbf{V}^{2}}
$$

and the density of states

$$
\rho(E)=N \rho_{\mathrm{W}}(E)=\frac{2 N}{\pi E_{0}^{2}}\left(E_{0}^{2}-E^{2}\right)^{1 / 2},
$$

which is the famous semicircle, $E_{0}=2\left(N V^{2}\right)^{1 / 2}$ being its 'radius'.

If the diagonal matrix elements fluctuate with $\overline{E_{k}}=0, \overline{E_{k}^{2}}=2 \mathbf{V}^{2}$, as in the Gaussian orthogonal ensemble, the result does not change for $N \gg 1$, since such fluctuations of the basis state energies are small compared with the full width $E_{0}$ of the eigenvalue spectrum.

The semicircular strength function also describes the spreading in a banded random Hamiltonian, where $V_{k m}$ are non-zero for $|k-m| \leqslant b$ only. In this case the size $N$ of the matrix in equation (44) must be replaced by the bandwidth $b$. This solution remains valid for a banded Hamiltonian with a non-zero increasing diagonal, as long as

$$
D b \ll\left(b \mathbf{V}^{2}\right)^{1 / 2},
$$

that is the mean basis-state energy spacing $D$ remains small (Wigner 1955, 1957).

\subsubsection{Breit-Wigner profile}

In the opposite case, $D b \gg\left(b \mathbf{V}^{2}\right)^{1 / 2}$, the increasing energy denominators reduce the mixing of distant basis states and make the strength function narrower. If we consider an infinite uniform Hamiltonian with evenly spaced diagonal matrix elements, $E_{m+1}-E_{m}=D$, the sum in equation (32) can be replaced by an integral over $\mathrm{d} E_{m} / D$. The mean-squared matrix element $\overline{V_{k m}^{2}}$ is independent of $k$ (as well as $m$ ), and the function $\Sigma_{k}(E)$ also does not depend on $k$. Equation (32) then becomes

$$
\Sigma(E)=\int \frac{\overline{V_{k m}^{2}}}{E-E_{m}-\operatorname{Re}[\Sigma(E)]-\mathrm{i} \operatorname{Im}[\Sigma(E)]} \frac{\mathrm{d} E_{m}}{D},
$$

which, after closing the integration in the upper half-plane of complex $E_{m}$, reduces to the contribution of the pole, $\Sigma(E)=-\mathrm{i} \pi \overline{V^{2}} / D$. The corresponding strength function is immediately obtained from equation (35) as

$$
\rho_{\mathrm{W}}(E, k)=\frac{1}{2 \pi} \frac{\Gamma}{\left(E-E_{k}\right)^{2}+\Gamma^{2} / 4},
$$

where

$$
\Gamma=2 \pi \overline{\mathbf{V}^{2} \rho}
$$

and $\rho=D^{-1}$ is the density of states.

The Breit-Wigner solution is in fact valid for matrices where $\overline{V_{k m}^{2}}$ as well as $\rho$ change over the matrix, as long as they do not change much on the scale of 
$\left|E_{k}-E_{m}\right| \sim \Gamma$. In particular, equations (47) and (48) are applicable to banded random matrices at $\left|E-E_{k}\right|<D b$, if $\overline{V_{k m}^{2}}=\mathbf{V}^{2}=$ constant within the band. The necessary condition for this is $\Gamma<D b$, or $\mathbf{V}^{2}<D^{2} b$, which is the opposite of equation (46).

\subsection{Criterion of equilibrium (ergodicity) of chaotic eigenvectors}

\subsubsection{Strong mixing}

Although the matrix elements $\mathbf{V}_{k m}$ fluctuate, the solution $\Sigma_{k}(E)$ of equation (32) is a smooth function of both $k$ and $E$, provided that the summation over $m$ averages out these fluctuations. To ensure this, the number of terms effectively contributing to the sum must be large. If the Hamiltonian matrix is 'full', that is $V_{k m} \neq 0$ for any nearby pair of states $k$ and $m$, as well distant pairs, the condition of strong mixing and weak fluctuations is $\mathbf{V}>D$, just opposite to that of perturbation theory.

In many-body systems with a two-body interaction between the particles the offdiagonal Hamiltonian matrix elements $V_{k m}$ couple only those shell-model basis states $k$ and $m$ (1) which differ by positions of no more than two particles. If the number of active particles in the system is greater than two, there are many zeros in the Hamiltonian matrix, that is, it becomes increasingly sparse. Denoting the mean spacing $\left|E_{k}-E_{m}\right|$ between the nearest basis states directly coupled by $V_{k m}$ by $d_{\mathrm{f}}$ $\left(d_{\mathrm{f}} \gg D\right)$, we can write the condition for averaging of fluctuations as

$$
\Gamma_{k} \gg d_{\mathrm{f}}
$$

since $\left|E-E_{m}\right| \lesssim \operatorname{Im}\left|\Sigma_{k}(E)\right|=\frac{1}{2} \Gamma_{k}$ determines the range of $m$ which effectively saturate the sum. The imaginary part of $\Sigma_{k}(E)$ can be estimated from equation (32) as a usual contribution of a pole in the complex energy plane, which gives

$$
\Gamma_{k}(E)=2 \pi \overline{V_{k m}^{2}} \rho_{\mathrm{f}}(E),
$$

where $\rho_{\mathrm{f}}(E)=d_{\mathrm{f}}^{-1}$ is the density of states directly coupled to $k$, and $\overline{V_{k m}^{2}}$ is the average over non-zero matrix elements only. The condition (49) then becomes

$$
\overline{V_{k m}^{2}}>d_{\mathrm{f}}^{2} \text {. }
$$

This means that, for the strength functions to be smooth, the interaction must be large, that is non-perturbative. In this case the eigenvectors contain large numbers of components. The number of (principal) components is in fact $N_{\mathrm{p}} \sim \Gamma_{k} / D$ rather than $\Gamma_{k} / d_{\mathrm{f}}$, since mixing of the basis states within the energy interval $\Gamma_{k}$ is complete (ergodicity). In this regime the components $C_{k}^{(i)}$ display Gaussian statistics, and the fluctuations in the strength function $\rho_{k}(E)$ are small (equilibrium).

When we reduce the ratio $V_{k m} / d_{\mathrm{f}}$ the fluctuations of $\Gamma_{k}$ increase and at $V_{k m}<d_{\mathrm{f}}$ the smooth self-consistent solution of equation (32) disappears. Indeed, in this case the sum in this equation is dominated by one term with a minimal energy denominator $E-E_{m} \sim d_{\mathrm{f}}$. The absence of a smooth solution for the shape of the eigenstates and the strength function does not mean that the number of principal components in the eigenstates is small. $\uparrow$ However, the distribution of the components is not ergodic; it has many 'holes' within the energy interval $2 \pi \overline{V_{k m}^{2}} \rho_{\mathrm{f}}(E)$, and the fluctuations of

$\dagger$ The number $N_{\mathrm{p}}$ of principal components in this case can be defined as the inverse participation ratio: $N_{\mathrm{p}}^{-1}=\sum_{k}\left(C_{k}^{(i)}\right)^{4}$. 
$C_{k}^{(i)}$ become large and non-Gaussian. Indeed, the structure of the eigenstate in this case is governed by an interplay of perturbation theory chains, that is series in $V_{k m} /\left(E_{k}-E_{m}\right)$, and a possibility of very small denominators in these chains owing to fluctuations of the intervals between basis state energy levels $\ddagger$ (Flambaum and Izrailev 1997a,b).

\subsubsection{The role of two-body correlations}

If the dynamics of a many-body system are governed by two-body interactions, as in equation (2), the Hamiltonian matrix elements $H_{k j}$ become, in a certain way, correlated, even when the underlying two-body matrix elements $V_{\alpha \beta \gamma \delta}$ are random (Flambaum et al. 1996a). These correlations affect the derivation of equation (32) and, strictly speaking, make this equation invalid.

This effect emerges in the fourth order of the perturbation theory, hence, let us consider the contractions in the fourth-order diagrams (22). In the first of them the intermediate many-body basis states $m$ differ from $k$ by the positions of at most two particles, $|m\rangle=a_{\alpha_{1}}^{\dagger} a_{\beta 1}^{\dagger} a_{\beta} a_{\alpha}|k\rangle$, that is the summation over states $m$ (or $l$ ) in fact involves moving all possible pairs $\alpha, \beta$ from the state $k$ into unoccupied orbitals $\alpha_{1}, \beta_{1}$. The corresponding matrix element in the Hamiltonian is $H_{m k}=V_{\alpha_{1} \beta_{1} \beta \alpha}$. Similarly, in the second diagram in equation (22), $|m\rangle=a_{\alpha_{1}}^{\dagger} a_{\beta_{1}}^{\dagger} a_{\beta} a_{\alpha}|k\rangle$, and $|l\rangle=a_{\gamma_{1}}^{\dagger} a_{\delta_{1}}^{\dagger} a_{\delta} a_{\gamma}|m\rangle$, that is the states $m$ and $l$ are obtained from $k$ and $m$ by moving the fermion pairs $\alpha \beta$ and $\gamma \delta$ into unoccupied orbitals $\alpha_{1}, \beta_{1}$ and $\gamma_{1} \delta_{1}$ respectively. Hence, the matrix elements involved are $H_{m k}=V_{\alpha_{1} \beta_{1} \beta \alpha}$ and $H_{l m}=V_{\gamma_{1} \delta} \delta \gamma$. In both contractions the same matrix element appears twice, which ensures that these contractions are non-zero and have the same order of summation.

A new feature due to the two-body interaction emerges in the third contraction and may now involve three different intermediate states:

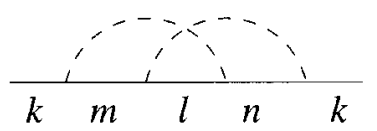

The numerator of the corresponding algebraic expression $H_{k n} H_{n l} H_{l m} H_{m k}$ contains four different Hamiltonian matrix elements. Nevertheless, its average is not zero, nor has it a lower order of summation than the other two fourth-order diagrams. Indeed, for $|m\rangle=a_{\alpha_{1}}^{\dagger} a_{\beta_{1}}^{\dagger} a_{\beta} a_{\alpha}|k\rangle$, and $|l\rangle=a_{\gamma_{1}}^{\dagger} a_{\delta_{1}}^{\dagger} a_{\delta} a_{\gamma}|m\rangle$, take $|n\rangle=a_{\alpha}^{\dagger} a_{\beta}^{\dagger} a_{\alpha_{1}} a_{\beta_{1}}|l\rangle$, which is equivalent to $|n\rangle=a_{\gamma_{1}}^{\dagger} a_{\delta 1}^{\dagger} a_{\delta} a_{\gamma}|k\rangle$. Then

$$
H_{k n} H_{n l} H_{l m} H_{m k}=V_{\gamma \delta \delta_{1} \gamma_{1}} V_{\alpha \beta \beta_{1} \alpha_{1}} V_{\gamma_{1} \delta_{1} \delta \gamma} V_{\alpha_{1} \beta_{1} \beta \alpha}
$$

and, in spite of the fact that all three intermediate states $m, n, l$ are different, the two two-body matrix elements $V_{\alpha_{1} \beta_{1} \beta \alpha}$ and $V_{\gamma_{1} \delta_{1} \delta \gamma}$ appear twice each in the contraction. Therefore, it cannot be discarded in the way that it has been in $\S 2.1$.

$\ddagger$ In most real systems with more then two particles the Hamiltonian matrix is sparse, that is it contains a certain fraction of zero off-diagonal matrix elements. In this case the non-zero $V_{k j}$ must be greater than the mean energy spacing between the directly coupled basis states (Altshuler et al. 1997, Jaquod and Shepelyansky 1997, Mirlin and Fyodorov 1997, Shepelyansky and Sushkov 1997). 
Another way to see the difference between a random Hamiltonian and that based on a two-body interaction is to consider the level density (11), which can be written as a trace

$$
\rho(E)=\operatorname{Tr}[\delta(E-H)]
$$

and its moments

$$
M_{m}=\int \rho(E) E^{m} \mathrm{~d} E=\operatorname{Tr}\left(H^{m}\right) .
$$

Suppose that the diagonal matrix elements are zeros (or have the same order of magnitude as the off-diagonal matrix elements and hence can be neglected when calculating the traces). The traces then have the same structure as the numerators of the perturbation theory expansion of $G_{k k}(20)$. All odd- $m$ traces vanish, signalling a symmetric level density. For a full uncorrelated random Hamiltonian the trace for an even $m=2 \nu$ is determined by the number of ways to arrange $\nu$ non-crossing contractions (known as a Catalan number)

$$
M_{2 \nu}=\frac{1}{\nu+1}\left(\begin{array}{c}
2 \nu \\
\nu
\end{array}\right) N^{\nu+1} V^{2 \nu},
$$

where $V^{2}=\overline{H_{k m}^{2}}$. These moments correspond to the 'semicircular' density (45) (for example Brody et al. (1981)).

On the other hand, if the Hamiltonian is generated by the two-body interactions, all binary contractions give equal contributions to the even moments (if we assume that the number of particles $n$ is large, and $n \gg m$, so that different pairs of orbitals dominate in the sums over the intermediate states). The even moments then are

$$
M_{2 \nu}=(2 \nu-1) ! ! N^{\nu+1} V^{2 \nu} .
$$

(Note that the second moments $M_{2}$ here and in equation (55) are the same and the difference emerges in the fourth order.) The moments (56) correspond to the Gaussian level density

$$
\rho(E)=\frac{N}{\left(2 \pi N \mathbf{V}^{2}\right)^{1 / 2}} \exp \left(-\frac{E^{2}}{2 N \mathbf{V}^{2}}\right),
$$

which is characteristic of the random two-body interaction model (Brody et al. 1981). A Gaussian level density is also a common feature of nuclear shell model calculations (Brody et al. 1981, Horoi et al. 1995a,b, Frazier et al. 1996, Zelevinsky et al. 1996) based on two-body Hamiltonians. These calculations are usually made in a restricted one- or two-shell model configuration space, and it obscures the role of the diagonal matrix elements $H_{k k}$ in forming the level density in the full problem. $\dagger$ On the other hand, if we apply equation (32) to a system with two-body interaction and degenerate or near-degenerate many-body basis states, the solution for the strength function and the density of states will come out in the form of a semicircle, and not a Gaussian.

$\dagger$ It is well known that the increase of the level density in a Fermi system, for example a nucleus or an atom, follows the law $\rho(E) \propto \exp \left(a E^{1 / 2}\right)$, derived for a non-interacting Fermi gas (Rosenzweig and Porter 1960, Bohr and Mottelson 1969, Camarda and Georgopulos 1983). 
However, there is sufficient evidence that the presence of an increasing diagonal in the Hamiltonian matrix restores the validity of equation (32). Physically, this is an effect of the mean field on the orbitals, as described by the one-body term in the Hamiltonian (2). The increase in spacings between the diagonal energies $E_{m}$ in the perturbation expansion (22) leads to the reduction in the higher-order contributions (where the two-body nature of the interaction makes a difference). The Breit-Wigner shape of the strength function (47) corresponding to $\Sigma(E)=-\mathrm{i} \pi \overline{V^{2}} / D$, is in fact given by the second-order contribution in $\Sigma$, and all higher-order terms in expansion (28) are equal to zero in this approximation. Additional evidence comes from numerical calculations for the random two-body interaction model (G. F. Gribakin 1999, unpublished). This was shown that even a small non-zero diagonal $H_{k k}$ generated by the one-body term in equation (2) quickly restores the applicability of equations (32)-(34) to the calculation of the strength functions and total density of states.

\section{$\S 3$. STATISTICAL DESCRIPTION}

\subsection{Microcanonical partition function}

In this section we are going to derive a partition function for a closed (isolated) system of a finite number of interacting particles. Examples of such systems are compound nuclei, complex atoms, atomic clusters and isolated quantum dots. This function allows one to perform analytical and numerical calculations of statistical mean values of different operators, for example occupation numbers.

Let us use the Hamiltonian (2) as a starting point. The one-body part incorporates the effect of a mean field, $\varepsilon_{\alpha}$ being the energies of the single-particle states (orbitals) in this field. The two-body part describes the residual interaction. For simplicity, here we neglect any dynamic effects of the interaction such as pairing and collective modes. Instead, we concentrate on the statistical effects of the interaction, therefore, in what follows we can assume that the matrix elements $V_{\alpha \beta \gamma \delta}$ are random variables.

The exact eigenstates of the Hamiltonian $|i\rangle$ are expressed in terms of the simple 'shell-model' basis states $|k\rangle$ by means of equation (5). They are characterized by their energies $E^{(i)}$. As discussed in $\S 2$, in complex systems they typically contain large numbers $N_{p} \gg 1$ of principal components $C_{k}^{(i)}$, which fluctuate 'randomly' as a function of indices $i$ and $k$.

Let us consider the occupation number $n_{\alpha}$ of a single-particle state $\alpha$ in a chaotic ('compound') eigenstate $|i\rangle$. It can be presented in terms of the eigenstate components as

$$
n_{\alpha}^{(i)}=\left\langle i\left|\hat{n}_{\alpha}\right| i\right\rangle=\sum_{k}\left|C_{k}^{(i)}\right|^{2}\left\langle k\left|\hat{n}_{\alpha}\right| k\right\rangle,
$$

where $\hat{n}_{\alpha}=a_{\alpha}^{\dagger} a_{\alpha}$ is the occupation number operator. Knowledge of the distribution of the occupation numbers enables one to calculate the mean value of any singleparticle operator $\langle\mathbf{M}\rangle=\sum_{\alpha} n_{\alpha} M_{\alpha \alpha}$. Moreover, the variance of the distribution of non-diagonal elements of $\mathbf{M}$ which describe transition amplitudes between chaotic eigenstates, can also be expressed through the occupation numbers $n_{\alpha}$ (Flambaum 1993, 1994, Flambaum et al. 1994, 1996a, Gribakina et al. 1995).

As one can see from equation (57), the mean values of occupation numbers depend on the shape of exact eigenstates, given by the 'spreading function' $F$ (in what follows, the $F$ function): 


$$
F_{k}^{(i)} \equiv \overline{\left|C_{k}^{(i)}\right|^{2}} \approx F\left(E_{k}, E^{(i)}\right) .
$$

This function is closely related to the strength function (7) and the density of states (11):

$$
\rho_{\mathrm{W}}(E, k) \approx F\left(E_{k}, E^{(i)}\right) \rho(E) .
$$

It follows from the consideration in $\S 2.1$ (see equation (35)) that the residual interaction $\mathbf{V}$ strongly mixes the basis states $k$ in the energy interval $\Gamma$ (spreading width) near the eigenstate energy $E^{(i)}$. Typically, this spreading function rapidly decreases with an increase in $\left|E_{k}-E^{(i)}\right|$ (since the admixture of distant component is small). In agreement with the theoretical considerations, recent numerical studies of the Cerium atom (Flambaum et al. 1994, Gribakina et al. 1995), the s-d nuclear shell model (Horoi et al. 1995a,b, Frazier et al. 1996, Zelevinsky et al. 1996) and random two-body interaction model (Flambaum et al. 1996a,b) showed that the typical shapes $F$ of exact eigenstates are almost the same in different many-body systems and have a universal form which essentially depends on the spreading width $\Gamma$. The latter can be expressed in terms of the parameters of the model (intensity $\mathbf{V}$ of the residual interaction, number $\eta$ of particles, excitation energy, etc,; see equation (50)). One can also measure the width of the $F$ function (58) via the number of principal components, $N_{p} \sim \Gamma / D$, where $D$ is the local mean level spacing between the compound states. In many-body systems the value of $D$ decreases exponentially with increase in the number of active (valence) particles. As a result, $N_{\mathrm{p}}$ is very large, about $10^{4}-10^{6}$ in excited (compound) nuclei and about $10^{2}-10^{5}$ in excited rare-earth or actinide atoms and many multicharged ions (Gribakin et al. 1999).

Using equations (57) and (58) and the normalization of the $F$ function $\sum_{k} F\left(E_{k}, E\right)=1$, we can write

$$
n_{\alpha}(E)=\frac{\sum_{k} n_{\alpha}^{(k)} F\left(E_{k}, E\right)}{\sum_{k} F\left(E_{k}, E\right)}
$$

where $n_{\alpha}^{(k)} \equiv\left\langle k\left|\hat{n}_{\alpha}\right| k\right\rangle$ equals 0 or 1 , depending on whether the orbital $\alpha$ is empty or occupied in the basis state $k$. This way of averaging the occupation numbers can be compared with microcanonical averaging, since it is defined for a fixed total energy $E$ of a system. In fact, equation (60) is equivalent to the introduction of a new kind of partition function

$$
Z(E)=\sum_{k} F\left(E_{k}, E\right),
$$

which is entirely determined by the shape of the chaotic eigenstates. In what follows, we term equation (60) the $F$ distribution.

Equation (60) gives a new insight into the problem of statistical description of complex systems. Indeed, as mentioned above, the shape of the $F$ function has universal features and can be often described analytically; therefore, in practice there is no need to diagonalize the huge Hamiltonian matrix of a many-body system in order to obtain statistical averages. Note that the summation in equation (60) is carried out over the unperturbed energies $E_{k}$ defined by the mean field, rather than over the exact eigenstates, as in the standard canonical distribution. As a result, the distribution of the occupation numbers can be derived analytically (see § 5) even for a few interacting particles, that is in the situation when the standard Fermi-Dirac distribution is not valid. 


\subsection{Transition to the canonical distribution}

It is instructive to compare our $F$ distribution (60) with the occupation numbers in an open system at a given temperature $T$ (e.g. a quantum dot with a fixed number of electrons). We can calculate the effect of non-diagonal matrix elements of the interaction on the distribution of the occupation numbers (the diagonal matrix elements are included in the definition of $E_{k}=H_{k k}$ ). This distribution is given by the canonical equation

$$
n_{\alpha}(T)=\frac{\sum_{i} n_{\alpha}^{(i)} \exp \left(-E^{(i)} / T\right)}{\sum_{i} \exp \left(-E^{(i)} / T\right)},
$$

where $T$ is the temperature and the index $i$ enumerates exact eigenstates. The important difference between the $F$ distribution (60) and the canonical distribution (62) is that in the former the occupation numbers are calculated for a specific energy $E$ of the system, while in the latter they correspond to a given temperature $T$. However, the results of calculations based on equations (62) and (57) can be compared with each other using the relation between the energy $E$ and the temperature $T$ :

$$
E=\langle E\rangle_{T}=\frac{\sum_{i} E^{(i)} \exp \left(-E^{(i)} / T\right)}{\sum_{i} \exp \left(-E^{(i)} / T\right)} .
$$

Let us substitute $n_{\alpha}^{(i)}$ from equation (57) into equation (62) and replace the summation over $i$ by integration over $\rho(E) \mathrm{d} E$ where $\rho(E)$ is the eigenvalue density:

$$
\sum_{i} n_{\alpha}^{(i)} \exp \left(-\frac{E^{(i)}}{T}\right) \approx \int n_{\alpha}(E) \Phi_{T}(E) \mathrm{d} E,
$$

where we have introduced the 'canonical (thermal) averaging' function,

$$
\Phi_{T}(E)=\rho(E) \exp \left(-\frac{E}{T}\right) .
$$

As a result, we can transform the canonical distribution (62) into a form similar to the $F$ distribution (60):

$$
n_{\alpha}(T)=\frac{\sum_{k} n_{\alpha}^{(k)} \tilde{F}\left(T, E_{k}\right)}{\sum_{k} \tilde{F}\left(T, E_{k}\right)},
$$

where the function $\tilde{F}\left(T, E_{k}\right)$ is the canonical average of $F$ :

$$
\begin{aligned}
\tilde{F}\left(T, E_{k}\right) & =\int F\left(E_{k}, E\right) \Phi_{T}(E) \\
& =\exp \left(-\frac{E_{k}}{T}\right) g_{k}(T)
\end{aligned}
$$

and

$$
g_{k}(T)=\int \rho_{\mathrm{W}}\left(z+E_{k}, k\right) \exp \left(-\frac{z}{T}\right),
$$

where $z=E-E_{k}$. This form of the canonical distribution which expresses the results in terms of the basis components (instead of exact eigenstates) may be convenient for the calculation of the occupation numbers and other mean values in quantum dots in thermal equilibrium with an environment (with no particle exchange). The occupa- 
tion numbers in the basis states are $n_{\alpha}^{(k)}=1$ or 0 , and it is very easy to perform the summation in equation (66) for $n_{\alpha}(T)$ or in equation (60) for $n_{\alpha}(E)$ numerically.

If the strength function $\rho_{\mathrm{W}}(E, k)$ is a function of $E-E_{k}$ only, that is we can neglect the separate dependence of $\Sigma_{k}(E)$ on the energy $E$ and index $k$ (see equation (35)), the function $g_{k}(T)$ becomes independent of the energy $E_{k}$ and index $k$. In this case $g(T)$ is a common factor which cancels out in the equation (66) for the occupation numbers. This is a surprising result, because it means that the distribution of the occupation numbers does not depend on the non-diagonal matrix elements of the interaction! The diagonal matrix elements of the interaction included in $E_{k}$ can still be important, especially in systems with a small number of active particles (see for example Flambaum et al. (1998b) who discussed the numerical calculation of the occupation numbers in the cerium atom with four electrons in the open shell). However, quite often we can strongly reduce the diagonal matrix elements of residual interaction by a proper choice of the mean-field potential (e.g. one can use the Hartree-Fock potential with temperature-dependent occupation numbers). In this case, one obtains the Fermi-Dirac (or Bose-Einstein) distribution of the occupation numbers with single-particle energies which may depend on temperature. Note that the 'initial' interaction between the particles is not assumed to be small!

There are several reasons why this conclusion is not applicable at low temperatures. If the excitation energy $E_{k}-E_{\text {min }}$ is smaller than the spreading width $\Gamma$, one has to take into account the energy-dependent limits of the integral in equation (68). The approximation of a constant shape of the strength function $\rho_{\mathrm{W}}$ may be valid for highly excited eigenstates only. The low-energy states described by the Breit-Wigner shape have the width $\Gamma_{k}(E)$ which may have a strong energy dependence. Finally, at very low excitation energies the condition of equilibrium $V_{k m}>d_{\mathrm{f}}$ is not satisfied, and there is no equilibrium distribution $F$. Therefore, for very low temperatures, equations (66) and (67) are not applicable. However, in this range there are good traditional approaches such as the Landau-Migdal Fermi-liquid theory for finite systems.

\subsection{Transition to the Fermi-Dirac distribution}

Let us now show how the standard Fermi-Dirac distribution emerges directly from the $F$ distribution (60) for a closed system, in the limit of large number of particles. By separating the sums over the states $k$ with $n_{\alpha}^{(k)}=0$ and $n_{\alpha}^{(k)}=1$, equation (60) can be rewritten as

$$
\begin{aligned}
n_{\alpha}(E) & =\frac{0+Z_{\alpha}\left(n-1, E-\tilde{\varepsilon}_{\alpha}\right)}{Z_{\alpha}\left(n-1, E-\tilde{\varepsilon}_{\alpha}\right)+Z_{\alpha}(n, E)} \\
& =\left(1+\frac{Z_{\alpha}(n, E)}{Z_{\alpha}\left(n-1, E-\tilde{\varepsilon}_{\alpha}\right)}\right)^{-1} .
\end{aligned}
$$

Here, two 'partial' partition functions $Z_{\alpha}(n, E)$ and $Z_{\alpha}\left(n-1, E-\tilde{\varepsilon}_{\alpha}\right)$ have been introduced. In the first $\left(n_{\alpha}^{(k)}=0\right)$, the summation is carried over all basis states of $n$ particles with the orbital $\alpha$ excluded: $Z_{\alpha}(n, E)=\sum_{k}^{\prime} F\left(E_{k}, E\right)$. The second sum $Z_{\alpha}\left(n-1, E-\tilde{\varepsilon}_{\alpha}\right)$ includes all basis states of $n-1$ particles with the orbital $\alpha$ excluded. The latter corresponds to the basis states in which the orbital $\alpha$ is filled $\left(n_{\alpha}^{(k)}=1\right)$; hence, the energy $\tilde{\varepsilon}_{\alpha} \equiv E_{k}(n)-E_{k}(n-1)$ of this orbital must be subtracted from the total energy $E$ of the $n$-particle system. (Here we in fact assume 
that the $F$ function depends on the difference $E_{k}-E$ only.) The energy $\tilde{\varepsilon}_{\alpha}$ can be obtained as

$$
\tilde{\varepsilon}_{\alpha}=\varepsilon_{\alpha}+\sum_{\beta \neq \alpha} U_{\alpha \beta} n_{\beta}^{(k)},
$$

where $\varepsilon_{\alpha}$ is the energy of a single-particle state and $U_{\alpha \beta} \equiv V_{\alpha \beta \beta \alpha}-V_{\alpha \beta \alpha \beta}$ is the diagonal matrix element of the two-body residual interaction (direct minus exchange) (see equation (2)). By considering $\tilde{\varepsilon}_{\alpha}$ to be independent of $k$ we assume that averaging over the basis states near the energy $E$ is possible. This assumption is equivalent to a local (at a given energy) mean-field approximation.

We should stress that this approximation is most important in deriving a good mean-field description from equation (2) for realistic systems. For example, in the cerium atom there are several orbitals belonging to different open subshells $(4 \mathrm{f}, 6 \mathrm{~s}$, etc.), which are quite close in energies and yet have very different radii. As a result, the Coulomb interactions between the electrons in such orbitals are very different (Flambaum et al. 1998b). In this case the second (interaction) term in equation (70) fluctuates strongly, depending on the occupation numbers of the other orbitals. As a result, there is no good mean-field approximation, and the equilibrium distribution of the occupation numbers is very different from the Fermi-Dirac distribution (Flambaum et al. 1998b). However, the $F$ distribution (60) gives a good description of the occupation numbers. In other cases, for example, the two-body random interaction model (Flambaum et al. 1996a,b, Flambaum and Izrailev 1997a,b) or the nuclear shell model (Horoi et al. 1995a,b, Frazier et al. 1996, Zelevinsky et al. 1996), the local mean-field approximation is quite accurate.

For a large number $n \gg 1$ of particles distributed over a large number $m \gg 1$ of orbitals, the dependence of $Z_{\alpha}$ on both $n$ and $\tilde{\varepsilon}_{\alpha}$ is very rapid, since the number $N$ of terms in the partition function $Z_{\alpha}$ is exponentially large: $N=m ! /[(m-n) ! n !]$. To make the dependence on the arguments smooth, we should consider $\ln Z_{\alpha}$ instead of $Z_{\alpha}$ and then expand

$$
\ln \left[Z_{\alpha}\left(n-\Delta n, E-\tilde{\varepsilon}_{\alpha}\right)\right] \approx \ln \left[Z_{\alpha}(n, E)\right]-A_{\alpha} \Delta n-B_{\alpha} \tilde{\varepsilon}_{\alpha},
$$

where

$$
A_{\alpha}=\frac{\partial\left(\ln Z_{\alpha}\right)}{\partial n}, \quad B_{\alpha}=\frac{\partial\left(\ln Z_{\alpha}\right)}{\partial E}, \quad \Delta n=1 .
$$

This immediately leads from equation (69) to a distribution of the Fermi-Dirac type:

$$
n_{\alpha}=\frac{1}{1+\exp \left(A_{\alpha}+B_{\alpha} \tilde{\varepsilon}_{\alpha}\right)} .
$$

If the number of essentially occupied orbitals in the definition of $Z_{\alpha}$ is large, the parameters $A_{\alpha}$ and $B_{\alpha}$ are not sensitive as to which particular orbital $\alpha$ is excluded from the sum and one can introduce $A_{\alpha}=A \equiv-\mu / T$, and $B_{\alpha}=B \equiv 1 / T$, as in the standard Fermi-Dirac distribution. The chemical potential $\mu$ and temperature $T$ are determined implicitly by the total number of particles and energy of the system:

$$
\begin{aligned}
& \sum_{\alpha} n_{\alpha}=n, \\
& \sum_{\alpha} \varepsilon_{s} n_{s}+\sum_{\alpha<\beta} U_{\alpha \beta} n_{\alpha} n_{\beta}=\frac{1}{2} \sum_{\alpha} n_{\alpha}\left(\varepsilon_{\alpha}+\tilde{\varepsilon}_{\alpha}\right)=E .
\end{aligned}
$$


Note that in equations (73) and (70), the terms containing the residual interaction $U_{\alpha \beta}$ can be substantially reduced by an appropriate choice of the mean-field basis (for instance, the terms $U_{\alpha \beta}$ may have different signs in such a basis). In practice, the values $\varepsilon_{\alpha}$ and $\tilde{\varepsilon}_{\alpha}$ can be very close. Since in equations (73) the non-diagonal matrix elements of the interaction are not taken into account, one can expect that the distribution of the occupation numbers defined by these equations gives correct results if the interaction is sufficiently weak (ideal-gas approximation). However, it can be shown (Flambaum and Izrailev 1997a,b) that even for strongly interacting particles the Fermi-Dirac distribution can be applied, if the total energy $E$ is corrected by taking into account the increase in temperature due to statistical effects of the interaction.

One should also note that a somewhat similar procedure transforms the canonical distribution (62) into the Fermi-Dirac distribution (for example Reif (1965)) in the case of many non-interacting particles (ideal gas). It is curious that the FermiDirac distribution is very close to the canonical distribution (62) even for a very small number of particles $(n=2)$, provided that the number of essentially occupied orbitals is large, that is for $T \gg \varepsilon$ or $\mu \gg \varepsilon$. In fact, this results from a large number of 'principal' terms in the partition function $Z_{\alpha}$, which enables one to replace $A_{\alpha}$ by $A$ in the ratio $Z_{\alpha}(n, T) / Z_{\alpha}(n-1, T) \equiv \exp \left(A_{\alpha}+B \varepsilon_{\alpha}\right)$ in the canonical distribution (62) (cf equation (69)).

A more accurate consideration shows, however, that the temperature $T$ in the Fermi-Dirac distribution is different from that in the canonical distribution. Indeed, using the expansion $A_{\alpha}=A\left(\varepsilon_{\mathrm{F}}\right)+A^{\prime}\left(\varepsilon_{\alpha}-\varepsilon_{\mathrm{F}}\right)$, where $\varepsilon_{\mathrm{F}}$ is the Fermi energy, one can obtain a relation between the Fermi-Dirac $\left(B_{\mathrm{FD}}\right)$ and canonical $(B)$ inverse temperatures: $B_{\mathrm{FD}}=B+A^{\prime} \varepsilon_{\mathrm{F}}$. The definition of the chemical potential also changes: $-\mu / T=A\left(\varepsilon_{\mathrm{F}}\right)-A^{\prime} \varepsilon_{\mathrm{F}}$. This is confirmed by numerical simulations for a closed system of few interacting Fermi particles (Flambaum et al. 1996a,b, Flambaum and Izrailev 1997a,b). The simulations show that, for the same total energy $E$ of the system, the canonical and Fermi-Dirac distributions give identical distributions $n_{\alpha}$. However, they correspond to different temperatures determined by equations (63) and (73), respectively.

The similarity of these two distributions for any number of particles is not so surprising in the presence of a thermal bath, where even a single particle is 'in equilibrium'. On the other hand, for closed systems with small numbers of particles the applicability of the Fermi-Dirac distribution is not obvious. To answer this question, one needs to analyse the role of interaction in the creation of an equilibrium distribution $(\S 2)$.

\section{§4. STATISTICAL DESCRIPTION OF TRANSITION AMPLITUDES}

\subsection{Mean-squared matrix elements}

Several approaches have been suggested to calculate matrix elements between compound states. Urin and Vyazankin (1991) expressed the mean-squared matrix element in terms of the strength function of a cold nucleus and calculated the latter semiempirically in the framework of 'temperature mechanism'. The calculations by Johnson et al. (1991), Johnson (1995) and Johnson and Bowman (1995) were based on the the work by French et al, (1988) and used the assumption that the meansquared matrix element of the parity-non-conserving interaction is proportional to that of the residual shell-model strong interaction. In this section we consider a 
statistical method described previously (Flambaum 1993, 1994, Flambaum and Vorov 1993, Flambaum et al. 1994, 1996a, Gribakina et al. 1995). It can be applied to calculation of mean-square matrix elements between chaotic (compound) states (5).

Let us consider a single-particle operator

$$
\hat{\mathbf{M}}=\sum_{\alpha \beta} a_{\alpha}^{\dagger} a_{\beta} M_{\alpha \beta}=\sum_{\alpha \beta} \rho_{\alpha \beta} M_{\alpha \beta} .
$$

It is convenient to express the matrix elements of $\hat{\mathbf{M}}$ in terms of matrix elements of the density matrix operator $\rho_{\alpha \beta}=a_{\alpha}^{\dagger} a_{\beta}$, which transfers a particle from orbital $\beta$ into orbital $\alpha$. One can see that the matrix element of $\hat{\mathbf{M}}$ between the compound states $n_{1}$ and $n_{2}$,

$$
\begin{aligned}
\left\langle n_{1}|\hat{\mathbf{M}}| n_{2}\right\rangle & =\sum_{\alpha \beta} M_{\alpha \beta}\left\langle n_{1}\left|\rho_{\alpha \beta}\right| n_{2}\right\rangle \\
& =\sum_{\alpha \beta} M_{\alpha \beta} \sum_{i j} C_{i}^{\left(n_{1}\right)}\left\langle i\left|\rho_{\alpha \beta}\right| j\right\rangle C_{j}^{\left(n_{2}\right)},
\end{aligned}
$$

has zero mean owing to the statistical properties of the components, that is $\overline{\left\langle n_{1}\left|\rho_{\alpha \beta}\right| n_{2}\right\rangle}=0$.

The variance of the matrix elements of $\hat{\mathbf{M}}$ can be presented in the following form:

$$
\overline{\left|M_{n_{1} n_{2}}\right|^{2}}=\sum_{\alpha \beta}\left|M_{\alpha \beta}\right|^{2} \overline{\left|\left\langle n_{1}\left|\rho_{\alpha \beta}\right| n_{2}\right\rangle\right|^{2}},
$$

where we have taken into account the result obtained by Flambaum et al. (1996a) that the average of the correlator

$$
\left\langle n_{1}\left|\rho_{\alpha \beta}\right| n_{2}\right\rangle\left\langle n_{2}\left|\rho_{\delta \gamma}\right| n_{1}\right\rangle=\sum_{i j k l} C_{i}^{\left(n_{1}\right)} C_{j}^{\left(n_{1}\right)} C_{k}^{\left(n_{2}\right)} C_{l}^{\left(n_{2}\right)}\left\langle i\left|\rho_{\alpha \beta}\right| k\right\rangle\left\langle l\left|\rho_{\delta \gamma}\right| j\right\rangle
$$

is zero, unless $\gamma=\alpha, \delta=\beta$. Therefore, the calculation of $\overline{\left|M_{n_{1} n_{2}}\right|^{2}}$, or a correlator $\overline{M_{n_{1} n_{2}} W_{n_{2} n_{1}}}$ is reduced to the calculation of $\overline{\left|\left\langle n_{1}\left|\rho_{\alpha \beta}\right| n_{2}\right\rangle\right|^{2}}$. The variance of the matrix element $\rho_{\alpha \beta}$ between the two compound states can be transformed into

$$
\begin{aligned}
\overline{\left|\rho_{\alpha \beta}^{\left(n_{1} n_{2}\right)}\right|^{2}} & =\overline{\left\langle n_{1}\left|\rho_{\alpha \beta}\right| n_{2}\right\rangle\left\langle n_{2}\left|\rho_{\beta \alpha}\right| n_{1}\right\rangle} \\
& =\overline{\sum_{i j k l} C_{i}^{\left(n_{1}\right)} C_{j}^{\left(n_{1}\right)} C_{k}^{\left(n_{2}\right)} C_{l}^{\left(n_{2}\right)}\left\langle i\left|\rho_{\alpha \beta}\right| k\right\rangle\left\langle l\left|\rho_{\beta \alpha}\right| j\right\rangle} \\
& =S_{\mathrm{d}}^{\left(n_{1} n_{2}\right)}+S_{\mathrm{c}}^{\left(n_{1} n_{2}\right)},
\end{aligned}
$$

where we separate the diagonal and non-diagonal contributions to the sum (78):

$$
\begin{aligned}
S_{\mathrm{d}}^{\left(n_{1} n_{2}\right)} & =\overline{\sum_{i k}\left|C_{i}^{\left(n_{1}\right)}\right|^{2}\left|C_{k}^{\left(n_{2}\right)}\right|^{2}\left|\left\langle i\left|\rho_{\alpha \beta}\right| k\right\rangle\right|^{2}}, \\
S_{\mathrm{c}}^{\left(n_{1} n_{2}\right)} & =\overline{\sum_{i \neq j, k \neq l} C_{i}^{\left(n_{1}\right)} C_{j}^{\left(n_{1}\right)} C_{k}^{\left(n_{2}\right)} C_{l}^{\left(n_{2}\right)}\left\langle i\left|\rho_{\alpha \beta}\right| k\right\rangle\left\langle l\left|\rho_{\beta \alpha}\right| j\right\rangle} .
\end{aligned}
$$

Note that the diagonal term $S_{\mathrm{d}}^{\left(n_{1} n_{2}\right)}$ is essentially positive. If the eigenstates are completely 'random' (different components both inside each eigenstate and of different eigenstates are uncorrelated), the correlation sum $S_{\mathrm{c}}$ is zero, and the variance 
is determined by the the diagonal sum $S_{\mathrm{d}}$ (this assumption was used in the previous calculations of matrix elements between the compound states (Sushkov and Flambaum 1982, Flambaum 1993, 1994, Flambaum and Vorov 1993, Flambaum et al. 1994, Gribakina et al. 1995)). However, as we show below, in many-body systems these two terms are of the same order, $S_{\mathrm{c}} \approx S_{\mathrm{d}}$, even if the two-body interaction $\mathbf{V}$ is random.

Following Flambaum et al. (1994) and Gribakina et al. (1995), let us replace the squared eigenstate components in equation (79) by their averages ( $F$ functions), equation (58). $\dagger$ The diagonal sum now takes the form

$$
S_{\mathrm{d}}^{\left(n_{1} n_{2}\right)}=\sum_{i k} F\left(E_{i}, E^{\left(n_{1}\right)}\right) F\left(E_{k}, E^{\left(n_{2}\right)}\right)\left\langle i\left|\rho_{\alpha \beta}\right| k\right\rangle\left\langle k\left|\rho_{\beta \alpha}\right| i\right\rangle .
$$

The summation over $k$ for a fixed $i$ includes only one state, $|k\rangle=\rho_{\beta \alpha}|i\rangle$, with $E_{k}=E_{i}+\omega_{\beta \alpha}$, where $\omega_{\beta \alpha}$ is the difference between the single-particle energies: $\omega_{\beta \alpha} \approx \varepsilon_{\beta}-\varepsilon_{\alpha}$. On the other hand, we can write

$$
\sum_{k}\left\langle i\left|\rho_{\alpha \beta}\right| k\right\rangle\left\langle k\left|\rho_{\beta \alpha}\right| i\right\rangle=\left\langle i\left|\rho_{\alpha \beta} \rho_{\beta \alpha}\right| i\right\rangle=\left\langle i\left|\hat{n}_{\alpha}\left(1-\hat{n}_{\beta}\right)\right| i\right\rangle,
$$

where $\hat{n}_{\alpha}=a_{\alpha}^{\dagger} a_{\alpha}$ and $\hat{n}_{\beta}=a_{\beta}^{\dagger} a_{\beta}$ are the occupation number operators. Thus, we obtain

$$
S_{\mathrm{d}}^{\left(n_{1} n_{2}\right)}=\sum_{i} F\left(E_{i}, E^{\left(n_{1}\right)}\right) F\left(E_{i}+\omega_{\beta \alpha}, E^{\left(n_{2}\right)}\right)\left\langle i\left|\hat{n}_{\alpha}\left(1-\hat{n}_{\beta}\right)\right| i\right\rangle .
$$

The matrix element $\left\langle i\left|\hat{n}_{\alpha}\left(1-\hat{n}_{\beta}\right)\right| i\right\rangle$ is equal to unity if the orbital $\alpha$ is occupied and $\beta$ is vacant in the basis state $|i\rangle$; otherwise, it is zero. Both $F$ values in equation (83) are smooth functions of energy normalized as $\sum_{i} F\left(E_{i}, E^{\left(n_{1}\right)}\right)=1$. This allows one to replace the matrix element of $\hat{n}_{\alpha}\left(1-\hat{n}_{\beta}\right)$ by its expectation value:

$$
\begin{aligned}
\overline{\left\langle i\left|\hat{n}_{\alpha}\left(1-\hat{n}_{\beta}\right)\right| i\right\rangle} & =\sum_{i} F\left(E_{i}, E^{\left(n_{1}\right)}\right)\left\langle i\left|\hat{n}_{\alpha}\left(1-\hat{n}_{\beta}\right)\right| i\right\rangle \\
& =\sum_{i} \overline{\left|C_{i}^{\left(n_{1}\right)}\right|^{2}}\left\langle i\left|\hat{n}_{\alpha}\left(1-\hat{n}_{\beta}\right)\right| i\right\rangle \\
& \approx\left\langle\hat{n}_{\alpha}\left(1-\hat{n}_{\beta}\right)\right\rangle_{n_{1}} .
\end{aligned}
$$

The sign $\approx$ above is a reminder that the left-hand side is the local average over the states $\left|n_{1}\right\rangle$. Practically, when the number of components is large, the fluctuations of $\left\langle\hat{n}_{\alpha}\left(1-\hat{n}_{\beta}\right)\right\rangle_{n_{1}}$ are expected to be small. Now we can rewrite equation (83) in the following form:

$$
S_{\mathrm{d}}^{\left(n_{1} n_{2}\right)}=\left\langle\hat{n}_{\alpha}\left(1-\hat{n}_{\beta}\right)\right\rangle_{n_{1}} \sum_{i} F\left(E_{i}, E^{\left(n_{1}\right)}\right) F\left(E_{i}+\omega_{\beta \alpha}, E^{\left(n_{2}\right)}\right) .
$$

It was shown in Flambaum et al. (1994) and Gribakina et al. (1995) that, under some reasonable assumptions about the $F$ functions, one can introduce a 'spread $\delta$ function' $\tilde{\delta}(\Delta)$,

$\dagger$ As usual, the averaging can be made either over a number of realizations of the two-body interaction matrix elements (ensemble average), or over a number of neighbouring eigenstates (physical energy average). In the spirit of ergodicity the results are assumed to be the same. 


$$
\begin{aligned}
\tilde{\delta}(\Delta) & =D_{2}^{-1} \sum_{i} F\left(E_{i}, E^{\left(n_{1}\right)}\right) F\left(E_{i}+\omega_{\beta \alpha}, E^{\left(n_{2}\right)}\right) \\
& =D_{2}^{-1} \int \frac{\mathrm{d} E_{i}}{D_{1}} F\left(E_{i}, E^{\left(n_{1}\right)}\right) F\left(E_{i}+\omega_{\beta \alpha}, E^{\left(n_{2}\right)}\right),
\end{aligned}
$$

where $\Delta=E^{\left(n_{2}\right)}-E^{\left(n_{1}\right)}-\omega_{\beta \alpha}$ and $D_{1}$ and $D_{2}$ are local mean level spacings for the $n_{1}$ and $n_{2}$ eigenstates. The function $\tilde{\delta}(\Delta)$ is symmetric, its characteristic width is determined by the spreading widths of the eigenstates $n_{1}$ and $n_{2}, \Gamma \approx \Gamma_{1}+\Gamma_{2}$, and it is normalized to unity, $\int \tilde{\delta}(\Delta) \mathrm{d} \Delta=1$, just as the standard $\delta$ function. If the $F$ values have Breit-Wigner shapes (see $\S 2$ ), $\tilde{\delta}$ is also a Breit-Wigner function:

$$
\tilde{\delta}(\Delta)=\frac{1}{\pi} \frac{\Gamma / 2}{\Delta^{2}+\Gamma^{2} / 4},
$$

with $\Gamma=\Gamma_{1}+\Gamma_{2}$. The fact that $S_{\mathrm{d}}^{\left(n_{1} n_{2}\right)}$ is proportional to the function $\tilde{\delta}(\Delta)$ is a particular manifestation of the energy conservation for transitions between the quasistationary basis states (Flambaum 1993, 1994, Flambaum and Vorov 1993) (if $\Gamma \rightarrow 0$, then $\tilde{\delta}(\Delta) \rightarrow \delta(\Delta)$ ). Using equations (76), (85) and (86) we can finally present the diagonal contribution to the variance of the matrix element $M_{n_{1} n_{2}}$ in the following form:

$$
\overline{\left|M_{n_{1} n_{2}}\right|_{\text {diag }}^{2}}=\sum_{\alpha \beta}\left|M_{\alpha \beta}\right|^{2}\left\langle\hat{n}_{\alpha}\left(1-\hat{n}_{\beta}\right)\right\rangle_{n_{1}} D_{2} \tilde{\delta}\left(E^{\left(n_{2}\right)}-E^{\left(n_{1}\right)}-\omega_{\beta \alpha}\right) .
$$

This expression is apparently asymmetric with respect to the states $n_{1}$ and $n_{2}$. By performing the calculation in a different way we can obtain

$$
S_{\mathrm{d}}^{\left(n_{1} n_{2}\right)}=\left\langle\hat{n}_{\beta}\left(1-\hat{n}_{\alpha}\right)\right\rangle_{n_{2}} \sum_{k} F\left(E_{k}-\omega_{\beta \alpha}, E^{\left(n_{1}\right)}\right) F\left(E_{k}, E^{\left(n_{2}\right)}\right)
$$

instead of equation (85), thereby arriving at a different formula for the variance:

$$
\overline{\left|M_{n_{1} n_{2}}\right|_{\text {diag }}^{2}}=\sum_{\alpha \beta}\left|M_{\alpha \beta}\right|^{2}\left\langle\hat{n}_{\beta}\left(1-\hat{n}_{\alpha}\right)\right\rangle_{n_{2}} D_{1} \tilde{\delta}\left(E^{\left(n_{2}\right)}-E^{\left(n_{1}\right)}-\omega_{\beta \alpha}\right) .
$$

In this form the occupation-number factor is calculated for the state $n_{2}$; it represents the probability of finding orbital $\beta$ occupied and $\alpha$ empty. If the assumptions made in the above derivations are correct, the two equations (88) and (90) should give identical results.

Previously (Flambaum et al. 1996a) we used the random two-body interaction model to check the accuracy of the statistical formulae derived above and found that the values of $S_{\mathrm{d}}^{\left(n_{1} n_{2}\right)}$ given by equations (85) and (89) almost coincided and were in good agreement with the initial expression (79). Similar tests were also made in the calculations for the cerium atom (Flambaum et al. 1994, Gribakina et al. 1995).

It is quite important for applications of the statistical approach (Flambaum 1993, 1994, Flambaum and Vorov 1993, Flambaum and Gribakin 1995, Flambaum et al. 1998a) that further simplifications be made by replacing the correlated occupancies product $\left\langle\hat{n}_{\alpha} \hat{n}_{\beta}\right\rangle_{n_{1}}$ in equation (84) by the product $\left\langle\hat{n}_{\alpha}\right\rangle_{n_{1}}\left\langle\hat{n}_{\beta}\right\rangle_{n_{1}}$ of the two mean values. This is definitely a valid operation when the numbers of excited particles and active orbitals are large, so that the occupation numbers for different orbitals become statistically independent. Then one would be able to use the following relation: 


$$
\left\langle\hat{n}_{\alpha}\left(1-\hat{n}_{\beta}\right)\right\rangle \approx n\left(\varepsilon_{\alpha}\right)\left[1-n\left(\varepsilon_{\beta}\right)\right],
$$

where $n\left(\varepsilon_{\alpha}\right)$ and $n\left(\varepsilon_{\alpha}\right)$ are the occupation numbers. This approximation was tested by Flambaum et al. (1996a) using the random two-body interaction model. Even for a system of four particles the accuracy of equation (91) remained reasonable, the error being about $10 \%$. This error is larger for the lowest eigenstates where the number of principal basis components is small and there is little or no thermalization and 'chaos'. In order to make a more direct test of the validity of equation (91), a correlator $\left\langle\hat{n}_{\alpha} \hat{n}_{\beta}\right\rangle_{n_{1}} /\left(\left\langle\hat{n}_{\alpha}\right\rangle_{n_{1}}\left\langle\hat{n}_{\beta}\right\rangle_{n_{1}}\right)$ was calculated as function of $n_{1}$. For a small number of particles $(n=4)$, this correlator displayed large fluctuations, however, its average value of about 0.8 was still rather close to 1 .

If the number of excited particles is large, one can use the Fermi-Dirac formula for the occupation numbers:

$$
n(\varepsilon)=\frac{1}{1+\exp [(\varepsilon-\mu) / T]}
$$

(see $\S 3.3$ ) with the temperature $T$ and chemical potential (Fermi energy) $\mu$ found from equations (73). Equations (88)-(91) together with equation (73) allow one to make computer calculations of the mean-squared matrix elements between compound states.

Following the same steps for a two-body operator

$$
\hat{\mathbf{U}}=\frac{1}{2} \sum_{\alpha \beta \gamma \delta} U_{\alpha \beta \gamma \delta} a_{\alpha}^{\dagger} a_{\beta}^{\dagger} a_{\gamma} a_{\delta},
$$

we obtain the following expression for the mean-squared matrix element:

$$
\overline{\left|U_{n_{1} n_{2}}\right|_{\text {diag }}^{2}}=\frac{1}{4} \sum_{\alpha \beta \gamma \delta}\left|U_{\alpha \beta \gamma \delta}-U_{\alpha \beta \delta \gamma}\right|^{2}\left\langle n_{\alpha} n_{\beta}\left(1-n_{\gamma}\right)\left(1-n_{\delta}\right)\right\rangle_{n_{1}} D_{2} \tilde{\delta}\left(\omega-\omega_{\gamma \delta, \alpha \beta}\right),
$$

where $\omega \equiv E^{\left(n_{2}\right)}-E^{\left(n_{1}\right)}$ and $\omega_{\gamma \delta, \alpha \beta}=\varepsilon_{\gamma}+\varepsilon_{\delta}-\varepsilon_{\alpha}-\varepsilon_{\beta}$ is the energy of the twoparticle transition $\alpha, \beta \rightarrow \gamma, \delta$.

We can also calculate the correlator $C_{\mathbf{M W}}$ between the matrix elements of two operators $\mathbf{M}$ and $\mathbf{W}$ with identical selection rules:

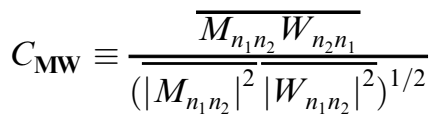

$$
\begin{aligned}
& =\frac{\sum_{\alpha \beta} M_{\alpha \beta} W_{\beta \alpha} \overline{\left|\rho_{\alpha \beta}^{\left(n_{1} n_{2}\right)}\right|^{2}}}{\left(\sum_{\alpha \beta}\left|M_{\alpha \beta}\right|^{2}\left|\rho_{\alpha \beta}^{\left(n_{1} n_{2}\right)}\right|^{2}\right)^{1 / 2}\left(\sum_{\alpha \beta}\left|W_{\alpha \beta}\right|^{2} \overline{\left.\rho_{\alpha \beta}^{\left(n_{1} n_{2}\right)}\right|^{2}}\right)^{1 / 2}} .
\end{aligned}
$$

One can easily see that $\left|C_{\mathbf{M W}}\right|=1$ if the matrix elements $W_{\alpha \beta}$ and $M_{\alpha \beta}$ are proportional to each other $\left(M_{\alpha \beta}=\right.$ constant $\left.\times W_{\alpha \beta}\right)$, or if there is only one dominating single-particle transition, say, $\mathrm{s} \rightarrow \mathrm{p}\left(M_{\mathrm{sp}} \gg M_{\alpha \beta}\right.$ and $W_{\mathrm{sp}} \gg W_{\alpha \beta}$ for all $\alpha \neq \mathrm{s}$, $\beta \neq \mathrm{p}$ ). Usually there are several important single-particle transitions near the Fermi surface $(q \approx 10)$. If there are no special reasons for the coherence or cancellations one may expect $\left|C_{\mathbf{M W}}\right| \approx 1 / q^{1 / 2} \approx 0.3$. However, in the most interesting case of $P$-odd and $P, T$-odd interactions there are pairs of opposite-sign contributions. Indeed, the matrix elements of the weak parity-non-conserving interaction are imaginary, $W_{\beta \alpha}=W_{\alpha \beta}^{*}=-W_{\alpha \beta}$, while the matrix elements of the $P, T$-odd interaction are real, $M_{\beta \alpha}=M_{\alpha \beta}$. Therefore, we have pairs of opposite-sign terms: 


$$
W_{\alpha \beta} M_{\beta \alpha}\left|\rho_{\alpha \beta}^{\left(n_{1} n_{2}\right)}\right|^{2}+W_{\beta \alpha} M_{\alpha \beta}\left|\rho_{\beta \alpha}^{\left(n_{1} n_{2}\right)}\right|^{2}=W_{\alpha \beta} M_{\beta \alpha}\left(\left|\rho_{\alpha \beta}^{\left(n_{1} n_{2}\right)}\right|^{2}-\left|\rho_{\beta \alpha}^{\left(n_{1} n_{2}\right)}\right|^{2}\right),
$$

which is proportional to $n_{\alpha}\left(1-n_{\beta}\right)-n_{\beta}\left(1-n_{\alpha}\right)$. This partial cancellation makes the correlator small $\left(\left|C_{\mathbf{M W}}\right| \leqslant 0.3\right)$, which means that in practice the matrix elements of the $P, T$-odd and the $P$-odd weak interactions are statistically independent.

Note that equations (88) and (94) for the matrix elements $M_{n_{1} n_{2}}$ and $U_{n_{1} n_{2}}$ respectively have a simple interpretation. The transition $\alpha \rightarrow \beta$ takes place if the orbital $\alpha$ is occupied and $\beta$ is vacant. Accordingly, the factor $n_{\alpha}\left(1-n_{\beta}\right)$ selects transitions near the Fermi surface. In the limit $\Gamma_{1}+\Gamma_{2} \rightarrow 0$, the function $\tilde{\delta}$ transforms into a conventional $\delta$ function. Therefore, it reflects the 'conservation of energy' for the quasistationary states with finite widths $\Gamma_{1}$ and $\Gamma_{2}$. The $\hat{\mathbf{M}}$ operator 'favours' the transitions between the compound states with the energy difference $E^{\left(n_{2}\right)}-E^{\left(n_{1}\right)} \equiv \omega$, close to $\omega_{\beta \alpha}$, where $\omega_{\beta \alpha}$ is the energy difference between the energies of the singleparticle orbitals coupled by $\hat{\mathbf{M}}$. In the case when $\omega \approx \omega_{\beta \alpha}$,

$$
\left(\overline{\left.\left|M_{n_{1} n_{2}}\right|^{2}\right)^{1 / 2}} \sim\left|M_{\alpha \beta}\right|\left(\frac{2 D_{2}}{\pi \Gamma}\right)^{1 / 2} \sim \frac{\left|M_{\alpha \beta}\right|}{N_{\mathrm{p} 2}^{1 / 2}},\right.
$$

where $M_{\alpha \beta}$ is the single-particle matrix element, and we have used equation (87) and assumed that the number $N_{\mathrm{p} 2}$ of principal components is large (much greater than unity). Far from 'resonance', at $\left|\omega-\omega_{\beta \alpha}\right|>\Gamma$ the matrix element is suppressed by the factor $\Gamma /\left|\omega-\omega_{\beta \alpha}\right|$ (in the Breit-Wigner model). This suppression is especially important for the calculations of the weak matrix element between nearby compound states in nuclei, where $\omega=0, \omega_{\beta \alpha} \approx 5-10 \mathrm{MeV} \geqslant \Gamma \approx 2 \mathrm{MeV}$.

\subsection{Correlations due to the two-body interaction}

In this section, following Flambaum et al. (1996a), we show how the basic twobody interaction gives rise to specific correlations between the Hamiltonian matrix elements, components of 'chaotic' eigenstates, and transition amplitudes.

Our consideration is based on the random two-body interaction model, originally introduced by French and Wong (1970) and Bohigas and Flores (1971). In this model, $n$ Fermi particles are distributed among $m$ non-degenerate orbitals with energies $\varepsilon$, coupled by random two-body matrix elements $V_{\alpha \beta \gamma \delta}$. The many-body basis states $|i\rangle$ are constructed by specifying the $n$ occupied orbitals. The energy $E_{i}$ of the basis state equals the sum of the single-particle energies over the occupied orbitals. The total number of the many-particle states in the model is $N=m ! /[n !(m-n) !] \sim \exp \{n \ln (m / n)+(m-n) \ln [m /(m-n)]\}$. The latter estimate relates to large $m$ and $n$ and shows that $N$ is exponentially large for $n, m-n \gg 1$.

The number of independent parameters of the many-body Hamiltonian is given by the number of different two-body interaction matrix elements $V_{\alpha \beta \gamma \delta}$ and equals $N_{2}=m^{2}(m-1)^{2} / 2$. Because of the two-body character of the interaction, the Hamiltonian matrix element $H_{i j}=\langle i|H| j\rangle$ is non-zero only when $|i\rangle$ and $|j\rangle$ differ by no more than two occupied single-particle orbitals. As a result, the number $K$ of the non-zero matrix elements $H_{i j}$ is given by

$$
K=N\left(K_{0}+K_{1}+K_{2}\right),
$$

where

$$
K_{0}=1, \quad K_{1}=n(m-n), \quad K_{2}=\frac{1}{4} n(n-1)(m-n)(m-n-1),
$$


are the numbers of the Hamiltonian matrix elements coupling a particular basis state $i$ to another basis state $j$ which differs from $i$ by the positions of no particles, one particle and two particles respectively. Therefore, for $n, m-n \gg 1$ we have $N_{2} \ll K \ll N^{2}$, that is the Hamiltonian matrix is essentially sparse and, in a sense, strongly correlated.

To see the correlation between non-zero matrix elements, let us consider a pair of basis states $|i\rangle$ and $|j\rangle$ which differ in the states of two particles, for example the state $|j\rangle$ can be obtained from the state $|i\rangle$ by transferring the particles from the orbitals $\alpha, \beta$ into the orbitals $\gamma, \delta$. For all such pairs, the Hamiltonian matrix elements are the same: $H_{i j}=V_{\alpha \beta \delta \gamma}$ (or, strictly speaking, $H_{i j}= \pm V_{\alpha \beta \delta \gamma}$, owing to Fermi statistics). It is easy to calculate the total number $N_{\text {eq }}$ of the matrix elements $H_{i j}$ equal to $V_{\alpha \beta \delta \gamma}$, using the fact that the remaining $n-2$ particles can be arbitrarily distributed over $m-4$ orbitals:

$$
N_{\text {eq }}=\frac{(m-4) !}{(n-2) !(m-n-2) !} .
$$

For basis states $|i\rangle$ and $|j\rangle$ which differ in the state of one particle $(\alpha \rightarrow \beta)$ the matrix element $H_{i j}$ equals the sum of the $n-1$ two-body interaction matrix elements, $H_{i j}=\sum_{\gamma} V_{\alpha \gamma \gamma \beta}$ (the index $\gamma$ runs over the rest $n-1$ occupied orbitals). In this case $H_{i j}$ for different $|i\rangle$ and $|j\rangle$ (with fixed $\alpha$ and $\beta$ ) do not coincide but may contain identical terms $V_{\alpha \gamma \gamma \beta}$, that is they are also correlated.

The eigenstates $\left|n_{1}\right\rangle$ of the model are determined by their components $C_{i}^{\left(n_{1}\right)}$ with respect to the many-particle basis states $|i\rangle$ and can be found by solving the Schrödinger equation:

$$
\sum_{j} H_{i j} C_{j}^{\left(n_{1}\right)}=E^{\left(n_{1}\right)} C_{i}^{\left(n_{1}\right)} .
$$

It is rather straightforward to show that the correlations between $H_{i j}$ result in correlations between the components $C_{i}^{\left(n_{1}\right)}$. Indeed, let us multiply the Schrödinger equation by the coefficient $C_{i}^{\left(n_{1}\right)}$ and sum over $n_{1}$. Using the orthogonality condition $\sum_{n_{1}} C_{i}^{\left(n_{1}\right)} C_{j}^{\left(n_{1}\right)}=\delta_{i j}$, one obtains

$$
H_{i j}=\sum_{n_{1}} C_{i}^{\left(n_{1}\right)} E^{\left(n_{1}\right)} C_{j}^{\left(n_{1}\right)} .
$$

In what follows, we assume that the matrix elements of the two-body interaction $\mathbf{V}$ are random variables with the zero mean, therefore, $\overline{H_{i j}}=0$ for $i \neq j$. In this case, one can obtain $\overline{C_{i}^{\left(n_{1}\right)} C_{k}^{\left(n_{1}\right)}}=0$ where the line stands for averaging over different realizations of $\mathbf{V}$. However, if matrix elements of the Hamiltonian are correlated, $\overline{H_{i j} H_{k l}} \neq 0$, the components of different eigenvectors $\left|n_{1}\right\rangle$ and $\left|n_{2}\right\rangle$ are also correlated, since

$$
\overline{H_{i j} H_{k l}}=\overline{\sum_{n_{1} n_{2}} C_{i}^{\left(n_{1}\right)} E^{\left(n_{1}\right)} C_{j}^{\left(n_{1}\right)} C_{k}^{\left(n_{2}\right)} E^{\left(n_{2}\right)} C_{l}^{\left(n_{2}\right)}} \neq 0 .
$$

The latter relation shows that $\overline{C_{i}^{\left(n_{1}\right)} C_{j}^{\left(n_{1}\right)} C_{k}^{\left(n_{2}\right)} C_{l}^{\left(n_{2}\right)}} \neq 0$.

This effect has important consequences. Below we show how these correlations emerge in the non-diagonal term $S_{\mathrm{c}}$ (equation (80)). First, note that for a given $i$ the sum over $k$ in equation (79) for $S_{\mathrm{d}}$ contains only one term, for which $|k\rangle=a_{\beta}^{\dagger} a_{\alpha}|i\rangle \equiv\left|i^{\prime}\right\rangle$, determined by transferring one particle from the orbital $\alpha$ to 
the orbital $\beta$ in the state $|i\rangle$ (hereafter we shall use the notation $i^{\prime}$ to denote such states). Accordingly, the index $i$ runs over those states in which $\alpha$ is occupied and $\beta$ is vacant. For such $i$ and $i^{\prime}$ the matrix element $\left\langle i\left|\rho_{\alpha \beta}\right| i^{\prime}\right\rangle=1$; otherwise, it is zero. Therefore, in fact, the sum in equation (79) is a single sum, with a number of items less than $N$ :

$$
S_{\mathrm{d}}^{\left(n_{1} n_{2}\right)}=\overline{\sum_{i}^{\prime}\left|C_{i}^{\left(n_{1}\right)}\right|^{2}\left|C_{i^{\prime}}^{\left(n_{2}\right)}\right|^{2}}
$$

where the sum $\sum_{i}{ }^{\prime}$ runs over the specified $i$. Analogously, equation (80) can be written as the double sum over $i$ and $j$ specified as above:

$$
S_{\mathrm{c}}^{\left(n_{1} n_{2}\right)}=\overline{\sum_{i \neq j}^{\prime \prime} C_{i}^{\left(n_{1}\right)} C_{j}^{\left(n_{1}\right)} C_{i^{\prime}}^{\left(n_{2}\right)} C_{j^{\prime}}^{\left(n_{2}\right)}},
$$

where $j^{\prime}$ is a function of $j,\left|j^{\prime}\right\rangle=a_{\beta}^{\dagger} a_{\alpha}|j\rangle$. Note that the energies of the basis states and their primed partners are connected as $E_{i^{\prime}}-E_{i}=\varepsilon_{\beta}-\varepsilon_{\alpha}=E_{j^{\prime}}-E_{j}$.

One can expect that maximal values of the sum (102) and, possibly, the sum (103) are achieved when the $C$ values are principal components of the eigenstates. This means that the mean square of the matrix element $\overline{\left|\left\langle n_{1}\left|\rho_{\alpha \beta}\right| n_{2}\right\rangle\right|^{2}}$ is maximal when the operator $\rho_{\alpha \beta}$ couples the principal components of the state $\left|n_{1}\right\rangle$ with those of $\left|n_{2}\right\rangle$, that is for $E^{\left(n_{1}\right)}-E^{\left(n_{2}\right)} \approx \omega_{\alpha \beta} \equiv \varepsilon_{\alpha}-\varepsilon_{\beta}$. Far from the maximum $\left(\left|E^{\left(n_{1}\right)}-E^{\left(n_{2}\right)}-\omega_{\alpha \beta}\right|>\Gamma\right)$ a principal component of one state, say, $n_{1}$, is coupled to a small component $k$ of the other state $n_{2}\left(\left|E_{k}-E^{\left(n_{2}\right)}\right|>\Gamma\right)$. The latter case is simpler to consider analytically, since the admixture of a small component in the eigenstate can be found by means of perturbation theory. This approach reveals the origin of the correlations in the sum $S_{\mathrm{c}}$ (equation (103)). For example, if $C_{j}^{\left(n_{1}\right)}$ is a small component of the eigenstate $n_{1}$, then it can be expressed as a perturbation theory admixture to the principal components. If $C_{i}^{\left(n_{1}\right)}$ is one of the latter, then there is a term in the sum (103), which is proportional to the principal component squared, $\left|C_{i}^{\left(n_{1}\right)}\right|^{2}$.

Indeed, there are four possibilities:

(i) $C_{i}^{\left(n_{1}\right)}$ and $C_{j^{\prime}}^{\left(n_{2}\right)}$ are among the principal components, and $C_{j}^{\left(n_{1}\right)}$ and $C_{i^{\prime}}^{\left(n_{2}\right)}$ correspond to the small components. Then, one can write

$$
\begin{aligned}
& C_{j}^{\left(n_{1}\right)}=\frac{\left\langle j|\mathbf{H}| \tilde{n}_{1}\right\rangle}{E^{\left(n_{1}\right)}-E_{j}}=\widetilde{\sum_{p}} \frac{H_{j p}}{E^{\left(n_{1}\right)}-E_{j}} C_{p}^{\left(n_{1}\right)}, \\
& C_{i^{\prime}}^{\left(n_{2}\right)}=\frac{\left\langle i^{\prime}|\mathbf{H}| \tilde{n}_{2}\right\rangle}{E^{\left(n_{2}\right)}-E_{i^{\prime}}}=\widetilde{\sum_{p}} \frac{H_{i^{\prime} q}}{E^{\left(n_{2}\right)}-E_{i^{\prime}}} C_{q}^{\left(n_{2}\right)} .
\end{aligned}
$$

The tilde above the summation sign indicates that the summation runs over the principal components only. The 'coherent' contribution to the sum $S_{\mathrm{c}}$ in equation (103) is obtained by separating the squared contributions of the principal components in the sums in $S_{\mathrm{c}}^{\left(n_{1} n_{2}\right)}$ (i.e. $\left.p=i, q=j^{\prime}\right)$ :

$$
\widetilde{\sum_{i, j^{\prime}}} \prime \prime \frac{\overline{H_{i^{\prime} j^{\prime}} H_{j i}}}{\left(E^{\left(n_{2}\right)}-E_{i^{\prime}}\right)\left(E^{\left(n_{1}\right)}-E_{j}\right)}\left|C_{i}^{\left(n_{1}\right)}\right|^{2}\left|C_{j^{\prime}}^{\left(n_{2}\right)}\right|^{2} .
$$


Taking into account that for the principal components we have $E_{i} \approx E^{\left(n_{1}\right)}$ and $E_{j^{\prime}} \approx E^{\left(n_{2}\right)}$, we can replace the energies, $E_{i^{\prime}} \rightarrow E^{\left(n_{1}\right)}+\omega_{\beta \alpha}$ and $E_{j} \rightarrow E^{\left(n_{2}\right)}-\omega_{\beta \alpha}$ and thus obtain the following contribution to $S_{\mathrm{c}}^{\left(n_{1} n_{2}\right)}$ :

$$
-\frac{1}{\left(E^{\left(n_{2}\right)}-E^{\left(n_{1}\right)}-\omega_{\beta \alpha}\right)^{2}} \widetilde{\sum_{i, j^{\prime}}}{ }^{\prime \prime}\left|C_{i}^{(1)}\right|^{2}\left|C_{j^{\prime}}^{(2)}\right|^{2} \overline{H_{i^{\prime} j^{\prime}} H_{i j}} .
$$

(ii) $C_{j}^{\left(n_{1}\right)}$ and $C_{i^{\prime}}^{\left(n_{2}\right)}$ correspond to the principal components, and $C_{i}^{\left(n_{1}\right)}$ and $C_{j^{\prime}}^{\left(n_{2}\right)}$ correspond to the small components. Then, the result is the same as equation (107).

(iii) $C_{i}^{\left(n_{1}\right)}$ and $C_{j}^{\left(n_{1}\right)}$ are principal components and $C_{i^{\prime}}^{\left(n_{2}\right)}$ and $C_{j^{\prime}}^{\left(n_{2}\right)}$ are small components (or (iv) $C_{i^{\prime}}^{\left(n_{2}\right)}$ and $C_{j^{\prime}}^{\left(n_{2}\right)}$ are principal components, and $C_{i}^{\left(n_{1}\right)}$ and $C_{j}^{\left(n_{1}\right)}$ are small components). In these cases there are no coherent terms in the sum for $S_{\mathrm{c}}$ in equation (80). This follows from the fact that for chaotic eigenstates the mixing among the principal components is almost complete, which makes them, to a good accuracy statistically independent.

Thus, far from the maximum, $\left|E^{\left(n_{2}\right)}-E^{\left(n_{1}\right)}-\omega_{\beta \alpha}\right|>\Gamma$, one obtains

$$
S_{\mathrm{c}}^{\left(n_{1} n_{2}\right)} \approx-\frac{2}{\left(E^{\left(n_{2}\right)}-E^{\left(n_{1}\right)}-\omega_{\beta \alpha}\right)^{2}} \widetilde{\sum_{i, j^{\prime}}}{ }^{\prime \prime}\left|C_{i}^{(1)}\right|^{2}\left|C_{j^{\prime}}^{(2)}\right|^{2} \overline{H_{i^{\prime} j^{\prime}} H_{i j}} .
$$

A similar calculation of the diagonal sum $S_{\mathrm{d}}^{\left(n_{1} n_{2}\right)}$ (equation (79)) yields

$$
\begin{aligned}
S_{\mathrm{d}}^{\left(n_{1} n_{2}\right)} \approx & \frac{1}{\left(E^{\left(n_{2}\right)}-E^{\left(n_{1}\right)}-\omega_{\beta \alpha}\right)^{2}} \\
& \times\left(\widetilde{\sum_{i}} \widetilde{\sum_{j^{\prime}}}\left|C_{i}^{\left(n_{1}\right)}\right|^{2}\left|C_{j^{\prime}}^{\left(n_{2}\right)}\right|^{2} \overline{H_{i^{\prime} j^{\prime}}^{2}}+\widetilde{\sum_{i}} \widetilde{\sum_{j^{\prime}}}{ }^{\prime}\left|C_{i}^{\left(n_{1}\right)}\right|^{2}\left|C_{j^{\prime}}^{\left(n_{2}\right)}\right|^{2} \overline{H_{i j}^{2}}\right) .
\end{aligned}
$$

The two terms in the large parentheses, result from the contribution of principal $i$ and small $i^{\prime}$ components in equation (102), and vice versa. From equation (108) we see that $S_{\mathrm{c}}^{\left(n_{1} n_{2}\right)}=0$ if $\overline{H_{i^{\prime} j^{\prime}} H_{i j}}=0$. However, there is nearly a $100 \%$ correlation between these matrix elements. Indeed, the basis state $i^{\prime}$ differs from $i$ by the location of only one particle (the transition from the orbital $\alpha$ to $\beta$ ), and the same is true for $j^{\prime}$ and $j$.

Let us estimate the relative magnitudes of $S_{\mathrm{d}}$ and $S_{\mathrm{c}}$. First, consider the case when $|i\rangle$ and $|j\rangle$ differ by two orbitals: $|j\rangle=a_{\nu_{2}}^{\dagger} a_{\mu_{2}}^{\dagger} a_{\mu_{1}} a_{\nu_{1}}|i\rangle$. In this case, $H_{i j}=V_{\nu_{1} \mu_{1} \mu_{2} \nu_{2}}$. Since the basis states $\left|i^{\prime}\right\rangle$ and $\left|j^{\prime}\right\rangle$ must differ by the same two orbitals, we have $H_{i^{\prime} j^{\prime}}=V_{\nu_{1} \mu_{1} \mu_{2} \nu_{2}}=H_{i j}$ (note that $\nu_{1}, \mu_{1}, \nu_{2}, \mu_{2} \neq \alpha, \beta$, since both states $|i\rangle$ and $|j\rangle$ contain $\alpha$ and do not contain $\beta$, whereas $\left|i^{\prime}\right\rangle$ and $\left|j^{\prime}\right\rangle$ contain $\beta$ and do not contain $\alpha$ ). Therefore, the averages over the non-zero matrix elements between such pairs of states are $\overline{H_{i j} H_{i^{\prime} j^{\prime}}}=\overline{H_{i j}^{2}}=\overline{H_{i^{\prime} j^{\prime}}^{2}}=\mathbf{V}^{2}$.

Now, let us consider the case when $|i\rangle$ and $|j\rangle$ differ by one orbital $|j\rangle=a_{\nu_{2}}^{\dagger} a_{\nu_{1}}|i\rangle$. In this case the Hamiltonian matrix elements are the sums of the $n-1$ two-body matrix elements, 


$$
\begin{aligned}
H_{i j} & =\sum_{\mu \neq \alpha}^{n-2} V_{\nu_{1} \mu \mu \nu_{2}}+V_{\nu_{1} \alpha \alpha \nu_{2}}, \\
H_{i^{\prime} j^{\prime}} & =\sum_{\mu \neq \beta}^{n-2} V_{\nu_{1} \mu \mu \nu_{2}}+V_{\nu_{1} \beta \beta \nu_{2}} .
\end{aligned}
$$

The sums of $n-2$ terms in $H_{i j}$ and $H_{i^{\prime} j^{\prime}}$ coincide; the difference is due to the one term only (orbital $\alpha$ is replaced by the orbital $\beta$ ). Thus,

$$
\begin{aligned}
\overline{H_{i j} H_{i^{\prime} j^{\prime}}} & =(n-2) \mathbf{V}^{2}, \\
\left(H_{i j}\right)^{2} & \overline{\left(H_{i^{\prime} j^{\prime}}\right)^{2}}=(n-1) \mathbf{V}^{2},
\end{aligned}
$$

where we took into account that $\overline{V_{\kappa \lambda \mu \nu} V_{\kappa_{1} \lambda_{1} \mu_{1} \nu_{1}}}=\mathbf{V}^{2} \delta_{\kappa \kappa_{1}} \delta_{\lambda \lambda_{1}} \delta_{\mu \mu_{1}} \delta_{\nu \nu_{1}}$.

The contributions of one-particle and two-particle transitions in equations (108) and (109) representing $S_{\mathrm{c}}$ and $S_{\mathrm{d}}$ respectively will be determined by the numbers of such transitions allowed by the corresponding sums. For the single-prime sums in equation (109) these numbers are proportional to $K_{1}$ and $K_{2}$ (equation (97)). In the double-prime sum in equation (108) these numbers are proportional to $\tilde{K}_{1}$ and $\tilde{K}_{2}$, the numbers of the two-body and one-body transitions $i \rightarrow j$, in the situation when one particle and the two orbitals ( $\alpha$ and $\beta$ ) do not participate in the transitions. These numbers can be obtained from equation (97) if we replace $n$ by $n-1$, and $m$ by $m-2$, so that $\tilde{K}_{1}=(n-1)(m-n-1)$, $\tilde{K}_{2}=(n-1)(n-2)(m-n-1)(m-n-2) / 4$. Finally we obtain that at $\left|E^{\left(n_{2}\right)}-E^{\left(n_{1}\right)}-\omega_{\beta \alpha}\right|>\Gamma$ the contribution of the correlation term to the variance of the matrix elements of $\rho_{\alpha \beta}$ can be estimated in the ratio as

$$
\begin{aligned}
R & \equiv \frac{S_{\mathrm{c}}}{S_{\mathrm{d}}} \\
& =-\frac{(n-2) \tilde{K}_{1}+\tilde{K}_{2}}{(n-1) K_{1}+K_{2}} \\
& =-\frac{(n-2)(m-n-1)(m-n+2)}{n(m-n)(m-n+3)} .
\end{aligned}
$$

This equation shows that for $n=2$ we have $S_{\mathrm{c}}=0$, which is easy to check directly, since $\left\langle H_{i^{\prime} j^{\prime}} H_{i j}\right\rangle=0$ in this case. For $n>2$ the correlation contribution $S_{\mathrm{c}}$ is negative at the tails of the strength distribution. This means that it indeed suppresses the transition amplitudes off-resonance. For $n, m-n \gg 1$ the ratio $R$ is approaching its limit value -1 . It is easy to obtain from equation (110) that, for $m-n \gg 1$,

$$
\frac{S_{\mathrm{d}}+S_{\mathrm{c}}}{S_{\mathrm{d}}}=1+R \approx \frac{2 m}{n(m-n)} .
$$

Thus, surprisingly, the role of the correlation contribution increases with increasing number of particles.

For $n=4, m=11(N=330)$, one obtains $R=-0.39$, which means that the correlation contribution reduces the magnitude of the squared matrix elements $\overline{\mathbf{M}^{2}}=\overline{\left|\rho_{\alpha \beta}^{(12)}\right|^{2}}$ between compound states almost by a factor of 2 (for $\left.\left|E^{\left(n_{2}\right)}-E^{\left(n_{1}\right)}-\omega_{\beta \alpha}\right|>\Gamma\right)$. The ratio found in numerical experiment is $R \approx-0.45$. 
We would like to stress that the role of the correlation term does not decrease with increases in the numbers of particles and orbitals. This prediction is supported by numerical experiment for $n=7$ and $m=14, N=3432$. The numerically found ratio is $R \approx-0.7$ versus $R=-0.55$ obtained from equation (111). The correlation contribution should be even more important in compound nuclei, where $N \approx 10^{5}$. This case can be modelled by the parameters $n=10, m=20$; then we have $R=-0.66$ or, equivalently, $\left(S_{\mathrm{d}}+S_{\mathrm{c}}\right) / S_{\mathrm{d}}=0.34$, which means that the correlations suppress the squared element $\mathbf{M}^{2}$ between compound states by a factor of three (far from its maximum).

It is worth emphasizing that the existence of correlations due to the perturbation theory admixtures of small components to the chaotic eigenstates, which leads to a non-zero value of $S_{\mathrm{c}}$ (equation (103)), is indeed non-trivial. For example, if one examines the summand of equation (103) as a function of $i$ and $j$, it would be hard to guess that the sum itself is essentially non-zero, since positive and negative values of $C_{i}^{\left(n_{1}\right)} C_{j}^{\left(n_{1}\right)} C_{i^{\prime}}^{\left(n_{2}\right)} C_{j^{\prime}}^{\left(n_{2}\right)}$ seem to be equally frequent and have roughly the same magnitude (Flambaum et al. 1996a).

Since $\sum_{n_{1}} S_{\mathrm{c}}^{\left(n_{1} n_{2}\right)}=\sum_{n_{2}} S_{\mathrm{c}}^{\left(n_{1} n_{2}\right)}=0$ (see below), the suppression of $\overline{\mathbf{M}}^{2}$ at the tails should be accompanied by correlational enhancement of the matrix elements near the maximum (at $\left|E^{(2)}-E^{(1)}-\omega_{\beta \alpha}\right|<\Gamma$ ). Thus, we come to the important conclusion that, even for a random two-body interaction, the correlations produce some sort of a 'correlation resonance' in the distribution of the squared matrix elements $\mathbf{M}^{2}$. One should note that this increase in the correlation effects in the matrix elements of a perturbation can be explained by the increased correlations between the Hamiltonian matrix elements when the number of particles and orbitals increases $(N / n \propto \exp n)$.

Now we can estimate the size of the correlation contribution $S_{\mathrm{c}}$ near the maximum of the the $\mathbf{M}^{2}$ distribution (at $\left|E^{\left(n_{2}\right)}-E^{\left(n_{1}\right)}-\omega_{\beta \alpha}\right|<\Gamma$ ). First, we show that, after summation over one of the compound states, the correlation contribution vanishes. Indeed,

$$
\begin{aligned}
\sum_{n_{2}} S_{\mathrm{c}}^{\left(n_{1} n_{2}\right)} & =\sum_{n_{2}} \sum_{i \neq j, k \neq l} C_{i}^{\left(n_{1}\right)} C_{j}^{\left(n_{1}\right)} C_{k}^{\left(n_{2}\right)} C_{l}^{\left(n_{2}\right)}\left\langle i\left|\rho_{\alpha \beta}\right| k\right\rangle\left\langle l\left|\rho_{\beta \alpha}\right| j\right\rangle \\
& =\sum_{i \neq j, k \neq l} C_{i}^{\left(n_{1}\right)} C_{j}^{\left(n_{1}\right)}\left\langle i\left|\rho_{\alpha \beta}\right| k\right\rangle\left\langle l\left|\rho_{\beta \alpha}\right| j\right\rangle \sum_{n_{2}} C_{k}^{\left(n_{2}\right)} C_{l}^{\left(n_{2}\right)} \\
& =0
\end{aligned}
$$

where we take into account that the sum over $n_{2}$ in the expression above is zero for $k \neq l$. Therefore, the negative value of $S_{\mathrm{c}}^{\left(n_{1} n_{2}\right)}$ at $\left|E^{\left(n_{2}\right)}-E^{\left(n_{1}\right)}-\omega_{\beta \alpha}\right|>\Gamma$ must be compensated by its positive value near the maximum. The sum rule (112) allows one to make a rough estimate of $S_{\mathrm{c}}$ near the maximum of $S_{\mathrm{d}}$ (and $\overline{\mathbf{M}^{2}}$ ).

Let us assume that $S_{\mathrm{c}}=R_{\mathrm{m}} S_{\mathrm{d}}$ at $\left|E^{\left(n_{2}\right)}-E^{\left(n_{1}\right)}-\omega_{\beta \alpha}\right|<\Gamma / 2$, whereas $S_{\mathrm{c}}=R_{\mathrm{t}} S_{\mathrm{d}}$ at $\left|E^{\left(n_{2}\right)}-E^{\left(n_{1}\right)}-\omega_{\beta \alpha}\right|>\Gamma / 2\left(R_{\mathrm{t}}\right.$ is given by equation (111)). The distribution of $S_{\mathrm{d}}^{\left(n_{1} n_{2}\right)}$ can be reasonably approximated by the Breit-Wigner shape (see $\S 2$ ):

$$
S_{\mathrm{d}}^{\left(n_{1} n_{2}\right)}=\frac{A}{E^{2}+\Gamma^{2} / 4},
$$

where $E=E^{\left(n_{2}\right)}-E^{\left(n_{1}\right)}-\omega_{\beta \alpha}$, and $\Gamma=\Gamma_{n_{1}}+\Gamma_{n_{2}}$. The sum rule (112) implies that 


$$
R_{\mathrm{m}} \int_{0}^{\Gamma / 2} \frac{\mathrm{d} E}{E^{2}+\Gamma^{2} / 4}+R_{\mathrm{t}} \int_{\Gamma / 2}^{\infty} \frac{\mathrm{d} E}{E^{2}+\Gamma^{2} / 4}=0
$$

Since the two integrals in the above equation are equal, we have $R_{\mathrm{m}}=-R_{\mathrm{t}}$. Thus, near the maximum the correlation contribution $S_{\mathrm{c}}$ is positive and enhances the squared matrix element with respect to the diagonal contribution:

$$
\begin{aligned}
\frac{S_{\mathrm{d}}+S_{\mathrm{c}}}{S_{\mathrm{d}}} & =1+R_{\mathrm{m}} \\
& =2-\left(1+R_{\mathrm{t}}\right) \\
& \approx 2\left(1-\frac{m}{n(m-n)}\right) .
\end{aligned}
$$

For larger $n$ and $m$ the correlation enhancement factor asymptotically reaches its maximal value of 2 . The numerical calculations for $n=4, m=11$, and $n=7$, $m=14$, show that the enhancement of $\overline{\mathbf{M}^{2}}$ with respect to $S_{\mathrm{d}}$ at the maximum is even greater in size than that predicted by equation (115). This is not too surprising since in equations (113)-(115) we estimated the average value of $R_{\mathrm{m}}$ over an interval $\Delta E \approx \Gamma$ around the maximum rather than the peak value at the maximum.

It is interesting that the correlations create a sharp spike-like form of the distribution, instead of a smooth Gaussian or Breit-Wigner form (see the details given by Flambaum et al. (1996a)). With such sharp peaks, the strength function for any particular operator $\hat{\mathbf{M}}$ can have the so-called gross-structure, owing to many single particle transition terms in equation (76). Without these specific correlations, the strength function would be much smoother and the gross structure would not be seen. It is also interesting to note that there are very large mesoscopic-type fluctuations in the distribution near the maximum, depending on a specific (random) realization of the two-body interaction $\mathbf{V}$. This is also the consequence of strong correlations.

A similar estimate of $S_{\mathrm{c}}$ near the maximum can be obtained by the direct calculation of the small component contribution to $S_{\mathrm{c}}$ (equation (103)). On an assumption that there are no correlations between principal components of compound states we can separate the contribution of small components. For example, in the resonance situation, $E^{\left(n_{2}\right)}-E^{\left(n_{1}\right)} \approx \omega_{\beta \alpha}$, if the components $S_{j}^{\left(n_{1}\right)}$ and $S_{j^{\prime}}^{\left(n_{2}\right)}$ are small $\left(\left|E_{j}-E^{\left(n_{1}\right)}\right|>\Gamma\right.$ and, consequently, $\left.\left|E_{j^{\prime}}-E^{\left(n_{2}\right)}\right|>\Gamma\right)$, then they contain contributions proportional to the principal components $C_{i}^{\left(n_{1}\right)}$ and $C_{i^{\prime}}^{\left(n_{2}\right)}$ (see equations (104) and (105)). Analogously, $S_{j}^{\left(n_{1}\right)}$ and $S_{j^{\prime}}^{\left(n_{2}\right)}$ may be among the principal components, and then the small components $C_{i}^{\left(n_{1}\right)}$ and $C_{i^{\prime}}^{\left(n_{2}\right)}$ will contain correlated contributions. Thus, we have the following estimate:

$$
S_{\mathrm{c}}^{\left(n_{1} n_{2}\right)} \approx 2 \widetilde{\sum_{p}} \sum_{\text {small }, j}\left|C_{i}^{\left(n_{1}\right)}\right|^{2}\left|C_{i^{\prime}}^{\left(n_{2}\right)}\right|^{2} \frac{\overline{H_{i j} H_{i^{\prime} j^{\prime}}}}{\left(E^{\left(n_{1}\right)}-E_{j}\right)\left(E^{\left(n_{2}\right)}-E_{j^{\prime}}\right)} .
$$

Since $E_{j^{\prime}}-E_{j}=E_{i^{\prime}}-E_{i} \approx E^{\left(n_{2}\right)}-E^{\left(n_{1}\right)}$ for the principal components $i$ and $i^{\prime}$, $E^{\left(n_{1}\right)}-E_{j}$ and $E^{\left(n_{2}\right)}-E_{j^{\prime}}$ in the denominator always have the same sign, and $S_{\mathrm{c}}$ is positive (recall that $\overline{H_{i j} H_{i^{\prime} j^{\prime}}}>0$ ). Equation (116) can be estimated using the spreading width $\Gamma=2 \pi \overline{H_{i j}^{2}} / D$, where $D$ is the mean level spacing for the many-body states. This yields $S_{\mathrm{c}} \approx S_{\mathrm{d}}$, in agreement with the previous estimate (115). 


\section{$\S 5$. CONCLUSIONS}

In this work we have formulated a statistical approach to isolated finite systems of interacting particles, which plays the same role as the canonical approach for systems in equilibrium with the thermal bath. It can be applied to complex manybody systems, for example compound nuclei, rare-earth and actinide atoms and multicharged ions, atomic clusters and quantum dots. The key point of this approach is a new kind of partition function defined by the shape of chaotic (compound) eigenstates ( $F$ function) with respect to the many-particle basis of a system without residual interaction (Slater determinants). This function is related to the strength function, which can be found from a set of equations obtained by statistical self-averaging in the system.

The $F$ distribution allows one to calculate mean values of different operators as a function of the total energy $E$ of the system. As an example, we have considered the occupation numbers $n_{\alpha}(E)$ and made comparisons with the standard canonical approach, which gives $n_{\alpha}(T)$, where $T$ is the temperature of an open system. In large systems (thermodynamic limit) the distribution of the occupation numbers $n_{\alpha}$ tends to the canonical distribution with the temperature $T^{-1}=\mathrm{d}(\ln \rho) / \mathrm{d} E$ where $\rho(E)$ is the energy level density.

Another important area of applications of our approach is the calculation of non-diagonal mean-squared matrix elements (transition amplitudes) between the the chaotic eigenstates in complex many-body systems. We show that the two-body nature of the interaction between the particles gives rise to specific correlations between the components of 'chaotic' compound eigenstates. These correlations, together with the correlations between the many-body Hamiltonian matrix elements, result in a relatively large correlation contribution to the mean-squared matrix element. The correlations exist even if the two-body matrix elements are independent random variables, for example in the random two-body interaction model. We demonstrate that the correlations can be understood in terms of the perturbative mixing of distant (small) components to the principal components of the eigenstates. If the Hamiltonian matrix elements are random variables the correlations of this type vanish.

One of the most interesting feature of the correlations found in our work is that they do not decrease with increase in the number of excited particles or active orbitals. In particular, this means that they must be taken into account in calculations of matrix elements of weak interactions between compound states in nuclei.

\section{ACKNOWLEDGEMENTS}

Many of the original results reported in this paper were obtained in close collaboration with G. Casati, A. A. Gribakina, F. M. Israilev, M. G. Kozlov, I. K. Ponomarev and O. K. Vorov. We are also grateful to O. P. Sushkov and V. G. Zelevinsky for valuable discussions. Support of this work by the Australian Research Council is gratefully acknowledged.

\section{REFERENCES}

Altshuler, B. L., Gefen, Yu., Kamenev, A., and Levitov, S. L., 1997, Phys. Rev. Lett., 78, 2803.

Bohigas, O., and Flores, J., 1971, Phys. Lett. B, 34, 261.

Bohr, A., and MotTelson, B., 1969, Nuclear Structure, Vol. 1 (New York: Benjamin). 
Brody, T. A., Flores, J., French, J. B., Mello, P. A., Pandey, A., and Wong, S. S. M., 1981, Rev. mod. Phys., 53, 385.

Camarda, H. S., and Georgopulos, P. D., 1983, Phys. Rev. Lett., 50, 492.

EdW ARdS, S. F., and WARner, M., 1980, J. Phys. A, 13, 381.

Flambaum, V. V., 1993, Physica scripta, 46, 198; 1994, Time Reversal Invariance and Parity Violation in Neutron Reactions, edited by C. R. Gould, J. D. Bowman and Yu. P. Popov (Singapore: World Scientific), p. 39.

Flambaum, V. V., and Gribakin, G.F., 1995, Prog. particle. nucl. Phys., 35, 423.

Flambaum, V. V., Gribakin, G. F., and Izrailev, F. M., 1996a, Phys. Rev. E, 53, 5729.

Flambaum, V. V., Gribakina, A. A., and Gribakin, G. F., 1998a, Phys. Rev. A, 58, 230.

Flambaum, V. V., Gribakina, A. A., Gribakin, G. F., and Kozlov, M. G., 1994, Phys. Rev. A, 50, 267.

Flambaum, V. V., Gribakina, A. A., Gribakin, G. F., and Ponomarev, I. V., 1998b, Phys. Rev. E, 57, 4933.

Flambaum, V. V., and Izrailev, F. M., 1997a, Phys. Rev. E, 55, R13; 1997b, ibid., 56, 5144.

Flambaum, V. V., Izrailev, F. M., and Casati, G., 1996b, Phys. Rev. E, 54, 2136.

Flambaum, V. V., and Vorov, O. K., 1993, Phys. Rev. Lett., 70, 4051.

Frazier, N., Brown, B. A., and Zelevinsky, V., 1996, Phys. Rev. C, 54, 1665.

French, J. B., Kota, V. K. B., PAndy, A., and Tomsovic, S., 1988, Ann. Phys. (NY), 181, 235.

French, J. B., and Wong, S. S. M., 1970, Phys. Lett. B, 35, 5.

Fyodorov, Y. V., Chubykalo, O. A., Izrailev, F. M., and Casati, G., 1996, Phys. Rev. Lett., 76, 1603.

Gribakin, G. F., Gribakina, A. A., and Flambaum, V. V., 1999, Aust. J. Phys., 52, 443.

Gribakina, A. A., Flambaum, V. V., and Gribakin, G. F., 1995, Phys. Rev. E, 52, 5667.

Horoi, M., Zelevinsky, V., and Brown, B. A., 1995a, Phys. Rev. Lett., 74, 5194; 1995b, Phys. Lett. B, 350, 141.

JaQuod, P., and ShePelyAnSKy, D. L., 1997, Phys. Rev. Lett., 79, 1837.

Johnson, M. B., 1995, Workshop on Parity and Time-Reversal Violation in Compound Nuclear States and Related Topics, Trento, Italy, 16-27 October.

Johnson, M. B., and Bowman, J. D., 1995, Phys. Rev. C, 51, 999.

Johnson, M. B., Bowman, J. D., and Yoo, S. H., 1991, Phys. Rev. Lett., 67, 310.

Mirlin, A. D., and Fyodorov, Y. V., 1997, Phys. Rev. B, 56, 13393.

PAStur, L. A., 1972, Teor. Mat. Fiz., 10, 102 (Engl. Transl., 1972, Theor. math. Phys., 10, 67); 1973, Russ. Math. Surveys, 28, 1.

REIF, F., 1965, Fundamentals of Statistical and Thermal Physics (Tokyo: McGraw-Hill Kogakusha).

Rosenzweig, N., and Porter, C.E., 1960, Phys. Rev., 120, 1698.

SHEPELYANSKY, D. L., and SUSHKOV, O. P., 1997, Europhys. Lett., 37, 121.

Sushkov, O. P., and Flambaum, V. V., 1982, Usp. Fiz. Nauk, 136, 3 (Engl. Transl., 1982, Soviet Phys. Usp., 25, 1).

Urin, M.H., and VyaZANKIN, O. N., 1991, Phys. Lett. B, 269, 13.

Wigner, E.P., 1955, Ann. Math., 62, 548; 1957, ibid., 65, 203.

Zelevinsky, V., Brown, B. A., FraZier, N., and Horoi, M., 1996, Phys. Rep., 276, 85. 